\title{
GASLESS COMBUSTION FRONTS WITH HEAT LOSS
}

\author{
ANNA GHAZARYAN, STEPHEN SCHECTER, AND PETER L. SIMON
}

\begin{abstract}
For a model of gasless combustion with heat loss, we use geometric singular perturbation theory to show existence of traveling combustion fronts. We show that the fronts are nonlinearly stable in an appropriate sense if an Evans function criterion, which can be verified numerically, is satisfied. For a solid reactant and exothermicity parameter that is not too large, we verify numerically that the criterion is satisfied.
\end{abstract}

\section{INTRODUCTION}

We consider the gasless combustion model

$$
\begin{aligned}
& \partial_{t} u_{1}=\partial_{x x} u_{1}+u_{2} \rho\left(u_{1}-\bar{u}_{1}\right)-\delta u_{1}, \\
& \partial_{t} u_{2}=\kappa \partial_{x x} u_{2}-\beta u_{2} \rho\left(u_{1}-\bar{u}_{1}\right),
\end{aligned}
$$

with $\beta>0, \delta \geq 0, \kappa \geq 0$, and

$$
\rho(u)= \begin{cases}e^{-\frac{1}{u}} & \text { if } u>0, \\ 0 & \text { if } u \leq 0 .\end{cases}
$$

Here $u_{1}$ is temperature, $u_{2}$ is reactant concentration, $u_{1}=\bar{u}_{1}$ is the temperature below which the reaction does not occur (ignition temperature), and $u_{1}=0$ is the ambient temperature. We assume that $\bar{u}_{1} \geq 0$, so the reaction does not occur at the ambient temperature. $\rho$ is the unit reaction rate, which is a function of temperature. Temperature, $x$, and $t$ have been scaled to normalize the first equation. $\beta$ is the exothermicity parameter; the larger $\beta$ is, the more fuel one must burn to achieve a given increase in temperature. $\kappa$ is diffusivity of the reactant, which is the inverse of the Lewis number; if $\kappa=0$, the reactant is a solid. The term $\delta u_{1}$ represents heat loss from the system to the environment according to Newton's law of cooling. The 4 -tuple $\left(\beta, \bar{u}_{1}, \delta, \kappa\right)$ is a vector of parameters.

Our interest is in traveling combustion fronts and their stability. Combustion fronts are a type of traveling wave. If they travel to the right, they connect a burned state at the left to an unburned state at the right. The unburned state is the state before combustion occurs; the temperature is 0 (ambient temperature), and the reactant concentration is positive. If there is no heat loss to the environment (i.e., if $\delta=0$ ), the burned state is at combustion temperature $\beta^{-1}$ and has reactant concentration 0 (all the reactant has burned). On the other hand, if there is heat loss to the environment $(\delta>0)$, the burned state cannot maintain a positive temperature; it, like the unburned state, is at the ambient temperature, due to

Date: January 6, 2013.

1991 Mathematics Subject Classification. 80A25, 35K57, 35B40, 34E15.

Key words and phrases. geometric singular perturbation theory, traveling wave, Evans function, stability.

This work was supported in part by the National Science Foundation under grants DMS-0908009 and DMS-0708386, and by OTKA under grant 81403 . 
gradual heat loss behind the combustion front. It turns out that in this case, some of the reactant remains unburned at the burned state. See Figure 1.1

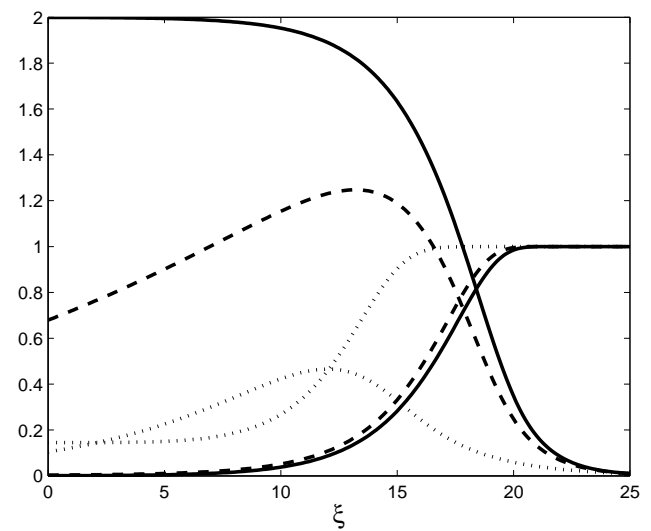

Figure 1.1. Combustion front profiles with $\beta=0.5$ and $\kappa=0$ (solid reactant). Curves that approach 1 (respectively 0) at the right are reactant concentration (respectively temperature). Solid: $\delta=0$. Behind the front, temperature is 2 (there is no heat loss to the environment) and all reactant has burned. Dashed: $\delta=0.0380$. Behind the front, temperature slowly falls due to heat loss to the environment. Dotted: $\delta=0.0561$. Behind the front, temperature again slowly falls. One can see more clearly than for $\delta=0.0380$ that there is unburned reactant behind the front.

The traveling combustion fronts of (1.1)-(1.2) have long been used for studies of premixed laminar flames, a basic topic in combustion theory; see the recent review article [21]. Questions of flame propagation and quenching translate into questions about existence and stability of traveling waves. In Section 2 we review the literature on existence and stability of combustion waves for the gasless combustion model, which often concerns various equivalent forms of the model.

We shall always limit our attention to fronts that approach both end states exponentially. This is only a limitation when $\delta=0$ and $\bar{u}_{1}=0$, i.e., when there is no heat loss to the environment and ignition temperature and ambient temperature are equal. Additional justifications for ignoring traveling waves that do not approach both end states exponentially have been given in the literature. For example, they vanish in the presence of heat loss to the environment, and they are only seen in simulations when carefully prepared, physically implausible initial conditions are used. There is a theoretical explanation for this observation; see $[8]$.

In this paper we do several things that add to the known facts about the gasless combustion model. In stating our results, and in the remainder of the paper, the reactant concentration at the unburned state is normalized to be 1 .

1. It is known that for $\delta=0$ (no heat loss) and small $\kappa \geq 0$ (small diffusivity of the reactant), the system (1.1)-(1.2) admits a unique combustion front with speed given by a smooth function $\sigma_{1}\left(\beta, \bar{u}_{1}, \kappa\right)$. In Section 3, we use geometric perturbation theory [13] to prove that these combustion fronts perturb to ones with small $\delta>0$. The wave speed $\sigma\left(\beta, \bar{u}_{1}, \delta, \kappa\right)$, with $\sigma\left(\beta, \bar{u}_{1}, 0, \kappa\right)=\sigma_{1}\left(\beta, \bar{u}_{1}, \kappa\right)$, is a smooth function. Previously this was known only numerically. 
We remark that here and throughout the paper, by a "unique combustion front" we mean unique up to shift; traveling waves can always be shifted in space, which corresponds to a shift of time.

2. It is known that for $\delta=0$ and small $\kappa \geq 0$, the unique combustion front is nonlinearly exponentially stable in an appropriate sense, provided an Evans function criterion is satisfied. In Section 4, we prove, for $\delta>0$, the corresponding result for the perturbations of these combustion fronts.

Let us give some background on this result. Denote the linearization of a PDE at a traveling wave by $u_{t}=L u$. We have the following notions:

- Spectral stability: the spectrum of $L$ is contained in $\operatorname{Re} \lambda \leq-\nu<0$, except for a simple eigenvalue $\lambda=0$, which traveling waves typically have because they can be shifted.

- Linearized stability: there is a space $Y$ of codimension one that is invariant under $e^{t L}$, and constants $\nu>0$ and $K \geq 1$, such that $\left\|e^{t L} \mid Y\right\| \leq K e^{-\nu t}$. ( $Y$ is complementary to the eigenspace of $L$ for the simple eigenvalue $\lambda=0$.)

- Nonlinear or orbital stability: for the nonlinear system, perturbations of the traveling wave stay close to the curve (in function space) of shifts of the traveling wave.

- Nonlinear exponential (respectively algebraic) stability with asymptotic phase: the nonlinear system is nonlinearly stable, and in addition, perturbations of the traveling wave decay exponentially (respectively algebraically) to a shift of the traveling wave.

These "definitions" are purposefully vague. Of course the space in which one calculates spectra must be taken into account. In some situations, perturbations of the traveling wave may be small in one space and satisfy the nonlinear stability or asymptotic stability conditions in another. However, a standard result, which does not apply to the gasless combustion model, is that if $L$ is sectorial and spectrally stable on a space $X$, then the traveling wave is linearly stable and nonlinearly exponentially stable with asymptotic phase on $X[19]$.

The essential spectrum of $L$ is determined by the linearization of the PDE at the end states of the wave. The discrete spectrum is found using the Evans function, an analytic function from $\mathbb{C}$ to $\mathbb{C}$. It is an infinite-dimensional analog of the characteristic polynomial, in that its zeros correspond to eigenvalues, and the multiplicity of the zero is the multiplicity of the eigenvalue [19].

The stability proof in Section 4 is nonstandard due to the fact that the essential spectrum of $L$ touches the imaginary axis, so $L$ is not spectrally stable. By using a weighted space one can move the essential spectrum to the left, but the spaces that achieve this are not closed under multiplication and so cannot easily be used to study the nonlinear problem. When $\kappa=0$ there is the additional difficulty that the essential spectrum includes a vertical line, so $L$ is not sectorial. We shall make use of machinery appropriate to dealing with these issues that was developed in [9] and [10], generalizing the methods of [8] (for gasless combustion with $\delta=0$ and $\kappa=0$ ) and [6] (for gasless combustion with $\delta=0$ and $\kappa>0$ ).

It is standard in applied mathematics to check for stability by checking that the spectrum of the linearization is contained in $\operatorname{Re} \lambda \leq 0$. Unfortunately, this condition alone does not imply anything stronger. For the gasless combustion model, using the machinery just mentioned allows one to pass from spectral calculations to detailed information about how perturbations of the combustion wave behave. 
More precisely, let $\mathcal{E}_{0}$ denote either $H^{1}(\mathbb{R})$ or $B U C$, the space of bounded uniformly continuous functions on $\mathbb{R}$ with the sup norm, and let $\mathcal{E}_{\alpha}$ denote the corresponding space with weight function $e^{\alpha \xi}, \xi=x-\sigma t$, where $\sigma$ is the velocity of the combustion front; $\alpha$ is positive and small. Functions in $\mathcal{E}_{\alpha}$ decay exponentially as $\xi \rightarrow \infty$ but may grow exponentially as $\xi \rightarrow-\infty$; functions in $\mathcal{E}_{0} \cap \mathcal{E}_{\alpha}$ decay exponentially as $\xi \rightarrow \infty$ and are bounded at the left. We show that as $t \rightarrow \infty$, perturbations of the combustion front that are small in $\mathcal{E}_{0} \cap \mathcal{E}_{\alpha}$ decay exponentially in $\mathcal{E}_{\alpha}$ to a shift of the combustion front. This is a type of nonlinear exponential stability with asymptotic phase. Furthermore, for $\delta>0$, the $u_{1}$-component of the perturbation (temperature) decays exponentially in $\mathcal{E}_{0}$, and the $u_{2}$-component of the perturbation (reactant concentration) stays small in $\mathcal{E}_{0}$. This is a type of nonlinear orbital stability. Moreover, if $\kappa>0$ and the perturbation is, in addition, in $L^{1}$, then the $u_{2}$-component of the perturbation stays small in $L^{1}$ and decays algebraically in $L^{\infty}$ (like $\left.t^{-\frac{1}{2}}\right)$.

For $\delta>0$ these results have the following interpretation. If the combustion front is perturbed in a way that is bounded or in $H^{1}$ at the left and decays exponentially at the right, the solution eventually looks like the combustion front at the right but not necessarily at the left. The temperature component of the perturbation rapidly decays in time; at the left this is because of heat loss to the environment. The reactant component of the perturbation may not decay in time at the left when $\kappa=0$; this is because at the left, the temperature is too low for it to burn. (Both components of the perturbation decay exponentially at the right in time in the weighted norm, because initially they decay exponentially in space at the right, and, relative to the front, they move left.) In addition, when $\kappa>0$ and the perturbation is, in addition, in $L^{1}$, the reactant component of the perturbation at the left does at least decay by diffusion as expected.

The behavior of temperature and reactant concentration at the left for $\delta>0$ is the reverse of their behavior for $\delta=0$; compare [8] and [6]. Without heat loss to the environment, the temperature behind the combustion front is high, so perturbations in the reactant concentration quickly decay (because the reactant burns), while perturbations in the temperature at best decay by diffusion.

3. In Section 5, we show analytically, for $\delta>0$, that the Evans function for the combustion front of Section 3 has a simple zero at the origin. This fact, which is equivalent to simplicity of the zero eigenvalue for the linearization of the system at the traveling wave, is needed for the stability result in Section 4. A similar result was obtained for $\delta=0$ in [8] and [7]. As in those papers, the result is a consequence of the nondegenerate splitting of invariant manifolds that occurs in the construction of the front.

4. Also in Section 5, for $\kappa=0, \beta$ not too large, and $\delta>0$, we provide numerical evidence that the Evans function for the combustion front of Section 3 has no zeros in $\operatorname{Re} \lambda \geq 0$, other than the simple zero at the origin. Similar numerical results have previously been obtained for the other cases; see the following section.

If we replace $u_{2} \rho\left(u_{1}\right)$ by a more general function $\omega\left(u_{1}, u_{2}\right)$, then, except in Section 5.3 where we do some numerics, the only properties of $\omega$ that are actually used are: $\omega$ is defined and $C^{3}$ on $\left\{\left(u_{1}, u_{2}\right): 0 \leq u_{2} \leq 1\right\} ; \omega\left(u_{1}, u_{2}\right)=0$ for $u_{1} \leq 0 ; \omega>0$ for $u_{1}>0$ and $u_{2}>0$; $\omega\left(u_{1}, 0\right)=0$ for all $u_{1}$; and $\frac{\partial \omega}{\partial u_{2}}\left(u_{1}, 0\right)>0$ for $u_{1}>0$. 


\section{Literature ON THE GASLESS COMBUSTION MODEL}

Instead of the combustion model (1.1)-(1.2), several equivalent forms are often used in the literature.

One equivalent form is

$$
\begin{aligned}
& \partial_{t} u_{1}=\partial_{x x} u_{1}+u_{2} \hat{\rho}\left(u_{1}-\bar{u}_{1}\right)-\delta u_{1}, \\
& \partial_{t} u_{2}=\kappa \partial_{x x} u_{2}-u_{2} \hat{\rho}\left(u_{1}-\bar{u}_{1}\right),
\end{aligned}
$$

where $\hat{\rho}$ is a function chosen from a one-parameter family of functions with properties similar to those of $\rho, \bar{u}_{1} \geq 0, \delta \geq 0$, and $\kappa \geq 0$. To derive (2.1)-(2.2) from (1.1)-(1.2), in (1.1)-(1.2) make the substitution $u_{1}=\frac{1}{\beta} \tilde{u}_{1}$. After dropping the tildes, one obtains (2.1)-(2.2) with $\hat{\rho}(u)=\beta \rho\left(\frac{u}{\beta}\right)$.

Another equivalent form is

$$
\begin{aligned}
& \partial_{t} u_{1}=\partial_{x x} u_{1}+u_{2} \epsilon^{-2} e^{\frac{1}{\epsilon}} \rho\left(\epsilon\left(u_{1}-\bar{u}_{1}\right)\right)-\hat{\delta} u, \\
& \partial_{t} u_{2}=\kappa \partial_{x x} u_{2}-u_{2} \epsilon^{-2} e^{\frac{1}{\epsilon}} \rho\left(\epsilon\left(u_{1}-\bar{u}_{1}\right)\right),
\end{aligned}
$$

with $\epsilon>0, \bar{u}_{1} \geq 0, \hat{\delta} \geq 0$, and $\kappa \geq 0$. Studying the limit $\epsilon \rightarrow 0$ is called high activation energy asymptotics. To derive (2.3)-(2.4) from (1.1)-(1.2), in (1.1)-(1.2) make the substitutions

$$
\beta=\epsilon^{-1}, \quad u_{1}=\epsilon \tilde{u}_{1}, \quad u_{2}=\tilde{u}_{2}, \quad t=\epsilon^{-1} e^{\frac{1}{\epsilon}} \tilde{t}, \quad x=\left(\epsilon^{-1} e^{\frac{1}{\epsilon}}\right)^{\frac{1}{2}} \tilde{x} .
$$

After dropping the tildes, one obtains (2.3)-(2.4) with $\hat{\delta}=\epsilon^{-1} e^{\frac{1}{\epsilon}} \delta$.

Remark 2.1. An alternative to (1.1)-(1.2) that is not equivalent to it is the system

$$
\begin{aligned}
& \partial_{t} u_{1}=\partial_{x x} u_{1}+u_{2} \rho\left(u_{1}\right)-\delta\left(u_{1}-u_{1}^{\dagger}\right), \\
& \partial_{t} u_{2}=\kappa \partial_{x x} u_{2}-\beta u_{2} \rho\left(u_{1}\right) .
\end{aligned}
$$

In this system, which is better physically motivated than (1.1)-(1.2), $u_{1}=0$ is absolute zero, and $u_{1}^{\dagger} \geq 0$ is ambient temperature. Unfortunately, if $u_{1}^{\dagger}>0$, physically meaningful traveling waves do not exist. This is called the cold boundary difficulty: traveling waves only exist when the ambient temperature is absolute zero. Nevertheless, approximate traveling waves have been studied numerically [12].

The system (2.5)-(2.6) is sometimes altered by replacing the Arrhenius reaction rate function $\rho\left(u_{1}\right)$ by the discontinuous function

$$
\rho_{\bar{u}_{1}}(u)= \begin{cases}e^{-\frac{1}{u}} & \text { if } u \geq \bar{u}_{1}, \\ 0 & \text { if } u<\bar{u}_{1},\end{cases}
$$

where $\bar{u}_{1}>0$ is ignition temperature. If $0 \leq u_{1}^{\dagger} \leq \bar{u}_{1}$, physically meaningful traveling waves often exist [1].

We shall now briefly review the literature on existence and stability of traveling combustion fronts for (1.1)-(1.2). As previously mentioned, we limit our discussion to fronts that approach their end states exponentially. Without loss of generality we restrict our attention to waves with positive velocity. 
2.1. Literature on traveling combustion fronts for (1.1)-(1.2) with no heat loss $(\delta=0)$. We consider fronts with values $\left(u_{1}, u_{2}\right)=\left(u_{1}^{*}, 0\right)$ at the left and $\left(u_{1}, u_{2}\right)=(0,1)$ at the right, where $u_{1}^{*}$ is the temperature of combustion. It must be determined; it turns out to be $\beta^{-1}$. We therefore assume $\bar{u}_{1}<\beta^{-1}$, i.e., ignition temperature is less than the temperature of combustion.

2.1.1. Existence and uniqueness. For infinite Lewis number $(\kappa=0)$ and $\bar{u}_{1}=0$, existence of a traveling front is shown in [4, 22]: the system reduces to one second-order equation by means of a first integral, and the proof is by phase plane analysis. The same proof would work for $0<\bar{u}_{1}<\beta^{-1}$. Uniqueness follows from the Melnikov integral calculation in [8]: the Melnikov integral is nonzero and has the same sign at any front. This material will be reviewed in Subsection 3.1. Earlier proofs using shooting were given in $[15,16]$. We denote the speed of the traveling front by $\sigma_{0}\left(\beta, \bar{u}_{1}\right)$; from the fact that the Melnikov integral is nonzero, it is a smooth function.

For finite Lewis number $(\kappa>0)$, there are the following existence and uniqueness results. The papers [3] and [17] use the system (2.1)-(2.2); we have converted their results into results for the system (1.1)-(1.2).

(1) $\kappa=1, \bar{u}_{1}=0$ : the system reduces to one second-order equation, and existence is proved by phase plane analysis [4]. The same proof would work for $0<\bar{u}_{1}<\beta^{-1}$. For $0<\bar{u}_{1}<\beta^{-1}$, uniqueness is shown in [3].

(2) $\kappa>0,0<\bar{u}_{1}<\beta^{-1}$ : proof of existence by Leray-Schauder degree [3]. For $0<\kappa<1$, uniqueness is shown in [17].

(3) $\kappa>0, \bar{u}_{1}=0$ : proof of existence by Leray-Schauder degree [17].

(4) $\kappa>0$ small, $\bar{u}_{1}=0$ : proof of existence and uniqueness by geometric singular perturbation theory [7]. The same proof would work for $0<\bar{u}_{1}<\beta^{-1}$. This method of proof also shows that the speed of the traveling wave for small $\kappa$ is close to that for $\kappa=0$, and in fact is a smooth function of $\left(\beta, \bar{u}_{1}, \kappa\right)$ for $\beta>0,0 \leq \bar{u}_{1}<\beta^{-1}$, and $0 \leq \kappa<\kappa_{0}\left(\beta, \bar{u}_{1}\right)$ for some positive function $\kappa_{0}$.

2.1.2. Stability. For $\bar{u}_{1}=0$, it is shown in [8] for $\kappa=0$ and in [6] for small $\kappa>0$ that the front is nonlinearly stable in an appropriate sense, provided the Evans function has no zeros in $\operatorname{Re} \lambda \geq 0$ other than a simple zero at the origin. The same proof would work for $0<\bar{u}_{1}<\beta^{-1}$. Simplicity of the zero at the origin is a consequence of the fact that the Melnikov integral mentioned above is nonzero; equivalently, it is a consequence of the nondegenerate splitting of invariant manifolds that occurs in the construction of the front.

Numerical computation with $\bar{u}_{1}=0$ indicates that the Evans function condition holds for $\beta$ not too large (for $\kappa=0$ see [8], for $\kappa>0$ see $[11,2]$ ). However, for larger $\beta$, the wave becomes unstable due to a pair of eigenvalues crossing into the right half-plane $[11,2]$.

2.2. Literature on traveling combustion fronts for $(1.1)-(1.2)$ with heat loss $(\delta>0)$. For $\delta>0$, spatially homogeneous equilibria of (1.1)-(1.2) all have temperature $u_{1}$ equal to 0 . We consider fronts with values $\left(u_{1}, u_{2}\right)=\left(0, u_{2}^{*}\right)$ at the left and $\left(u_{1}, u_{2}\right)=(0,1)$ at the right, where $u_{2}^{*}$, the concentration of unburned reactant behind the front, must be determined.

2.2.1. Infinite Lewis number $(\kappa=0)$. We found no results in the literature. 
2.2.2. Finite Lewis number $(\kappa>0)$. Existence:

(1) For the system (2.3)-(2.4) with $\epsilon$ small, $\kappa=1$, and $\bar{u}_{1}>0$ : proof of existence of two solutions for small $\hat{\delta}$ by Leray-Schauder degree [5].

(2) For the system (1.1)-(1.2) with $\beta=1, \kappa>0$, and $\bar{u}_{1}>0$ : proof of existence of two solutions for small $\delta$ by Leray-Schauder degree [18].

Numerical results: Figure 5 in [21], which uses the system (2.3)-(2.4), is typical of the numerical results in the literature. For $\left(\epsilon, \bar{u}_{1}\right)=(0.1,0)$, it shows the wave speed plotted against $\hat{\delta}$ for different values of $\kappa>0$. For $\hat{\delta}$ small there two traveling waves; they meet in a saddle-node bifurcation at some $\hat{\delta}=\hat{\delta}_{0}$, and there are no traveling waves for $\hat{\delta}>\hat{\delta}_{0}$. As $\hat{\delta} \rightarrow 0$, the two speed of one traveling wave appears to approach the speed of the combustion front for $\hat{\delta}=0$, and the speed of the other traveling wave appears to approach 0 .

Spectral stability: see the review article [21], which uses the form (2.3)-(2.4).

(1) Essential spectrum: At both ends of the wave, the spectrum of the corresponding constant-coefficient operator lies in the left half-plane but touches the imaginary axis. See Figure 6 in [21].

(2) Discrete spectrum: Determined using the Evans function. A typical result is shown in Figure 9 in [21], reproduced here as Figure 2.1. The figure shows, for $\left(\epsilon, \bar{u}_{1}, \kappa\right)=$ $(0.1,0,0.5)$, the curve in $\hat{\delta} \hat{\sigma}$-space $(\hat{\sigma}=$ wave speed $)$ for which traveling waves exist. On the upper part of the curve $(\hat{\delta}$ small, $\hat{\sigma}$ near the speed of the traveling wave for $\hat{\delta}=0$ ) all eigenvalues except for 0 lie in the left half-plane. At a point marked $H$ on the upper part of the curve a pair of complex eigenvalues crosses into the right half-plane. Thereafter the traveling waves are unstable. However, as one moves along the curve, these eigenvalues become real, and then, at the saddle-node bifurcation point (see p. 27), one of these real eigenvalues crosses back over the imaginary axis.

Figure 5 in [21] shows how this bifurcation diagram changes as $\kappa$ varies with $\epsilon$ held fixed. As $\kappa$ increases ( $L_{A}$ decreases), $H$ and the saddle-node bifurcation point come together in a Takens-Bogdanov point. After that the saddle-node bifurcation point is still present but $H$ is not. Above the saddle-node bifurcation point all eigenvalues except for 0 lie in the left half-plane, and at the saddle-node bifurcation point, one crosses into the right-half plane.

On the other hand, as $\kappa$ decreases ( $L_{A}$ increases), eventually $H$ moves left to $\hat{\delta}=0$. For lower $\kappa$ (higher $L_{A}$ ) all traveling waves are unstable.

For $\epsilon$ near 0 , this whole picture can be derived asymptotically [21].

The fact that for $\epsilon=0.1$ and small $\kappa$, all traveling waves are unstable, is consistent with [11], in which it is found that for $\delta=0$ and $\kappa$ small, the combustion front, which is stable for small $\beta$, loses stability in a Hopf bifurcation when $\beta$ increases past about 7 (or when $\epsilon$ fall below about 0.14 ).

For $\beta$ below about 7 and $\kappa$ small, the traveling wave is stable for $\delta=0$, and, as in Figure 2.1, we would find stability on the upper branch, at least near $\delta=0$.

It appears that for all $(\beta, \kappa)$ that have been studied, the traveling waves with $\delta$ below the saddle-node bifurcation value are unstable.

Linearized and nonlinear stability: not discussed in the literature. Since the essential spectrum of the linearization touches the imaginary axis, the spectral information just discussed does not imply either linearized or nonlinear stability. 


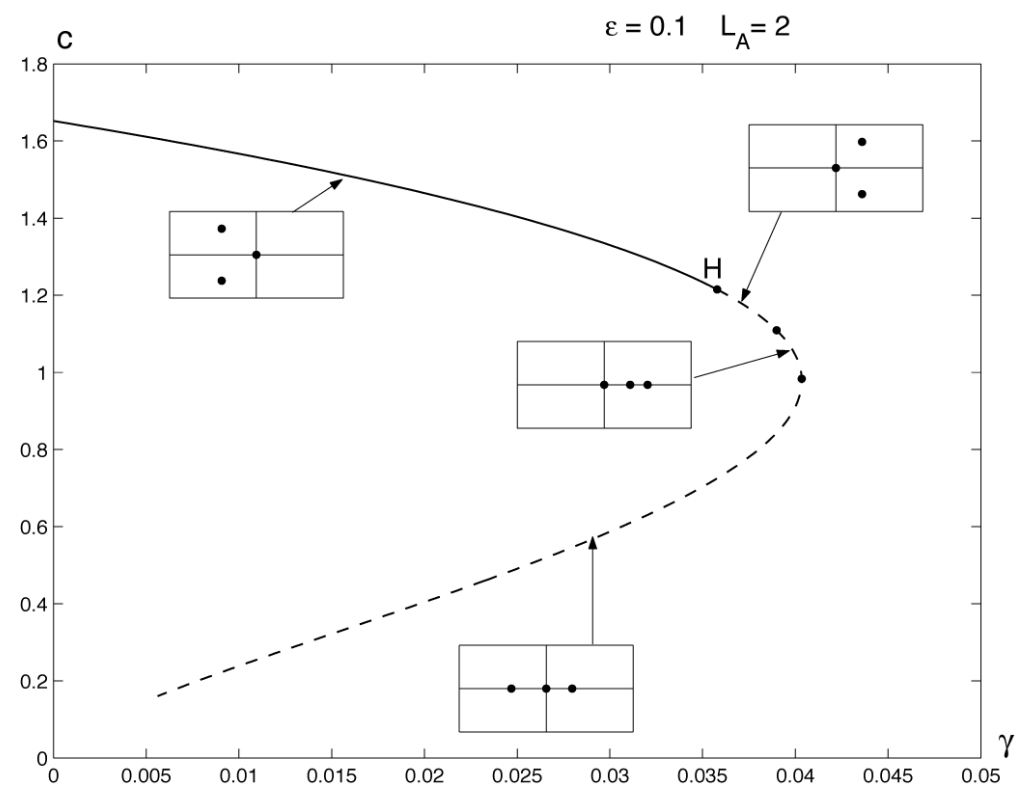

Figure 2.1. Figure 9 of [21], showing, for the system (2.3)-(2.4) with $\epsilon=0.1$, $\bar{u}_{1}=0$, and $\kappa=L_{A}^{-1}=0.5$, the curve in $\hat{\delta} \hat{\sigma}$-space $(\hat{\sigma}=$ wave speed $)$ for which traveling waves exist. In the figure, $\gamma$ corresponds to our $\hat{\delta}$ and $c$ to our $\hat{\sigma}$.

\section{Existence of traveling WaVes With SPEEd of order one}

In (1.1)-(1.2), we replace the spatial coordinate $x$ with one $\xi$ that is moving with velocity $\sigma: \xi=x-\sigma t$. We obtain

$$
\begin{aligned}
& \partial_{t} u_{1}=\partial_{\xi \xi} u_{1}+\sigma \partial_{\xi} u_{1}+u_{2} \rho\left(u_{1}-\bar{u}_{1}\right)-\delta u_{1} \\
& \partial_{t} u_{2}=\kappa \partial_{\xi \xi} u_{2}+\sigma \partial_{\xi} u_{2}-\beta u_{2} \rho\left(u_{1}-\bar{u}_{1}\right) .
\end{aligned}
$$

A steady solution of (3.1)-(3.2) is a traveling wave solution of (1.1)-(1.2) with velocity $\sigma$. Steady solutions of (3.1)-(3.2) satisfy the system of ODEs

$$
\begin{aligned}
& 0=\partial_{\xi \xi} u_{1}+\sigma \partial_{\xi} u_{1}+u_{2} \rho\left(u_{1}-\bar{u}_{1}\right)-\delta u_{1}, \\
& 0=\kappa \partial_{\xi \xi} u_{2}+\sigma \partial_{\xi} u_{2}-\beta u_{2} \rho\left(u_{1}-\bar{u}_{1}\right) .
\end{aligned}
$$

3.1. Infinite Lewis number $(\kappa=0)$, no heat loss $(\delta=0)$. This subsection should be regarded as a review.

We consider (3.3)-(3.4) with $\left(\beta, \bar{u}_{1}\right)$ fixed and $\delta=\kappa=0$. We are interested in solutions with $\sigma>0$ that satisfy the boundary conditions

$$
\left(u_{1}, \partial_{\xi} u_{1}, u_{2}\right)(-\infty)=\left(u_{1}^{*}, 0,0\right), \quad\left(u_{1}, \partial_{\xi} u_{1}, u_{2}\right)(\infty)=(0,0,1) .
$$

The temperature of combustion $u_{1}^{*}>\bar{u}_{1}$ and the speed $\sigma>0$ are yet to be determined. In addition we require that the solution approach its end states exponentially. As mentioned in the introduction, this is only a limitation when $\delta=0$ and $\bar{u}_{1}=0$. 
In the system (3.3)-(3.4) with $\delta=\kappa=0$, we set $v_{1}=\partial_{\xi} u_{1}$ and use prime to denote derivative with respect to $\xi$. We obtain the first-order system

$$
\begin{aligned}
& u_{1}^{\prime}=v_{1}, \\
& v_{1}^{\prime}=-\sigma v_{1}-u_{2} \rho\left(u_{1}-\bar{u}_{1}\right), \\
& u_{2}^{\prime}=\frac{\beta}{\sigma} u_{2} \rho\left(u_{1}-\bar{u}_{1}\right),
\end{aligned}
$$

which has the vector of parameters $\left(\beta, \bar{u}_{1}, \sigma\right)$. A solution of (3.3)-(3.4) that satisfies the boundary conditions (3.5) corresponds to a solution of (3.6)-(3.8) that goes from an equilibrium $\left(u_{1}^{*}, 0,0\right)$ to the equilibrium $(0,0,1)$.

The set of equilibria of (3.6)-(3.8) is the half plane $H_{\bar{u}_{1}}=\left\{\left(u_{1}, v_{1}, u_{2}\right): u_{1} \leq \bar{u}_{1}\right.$ and $v_{1}=$ $0\}$ together with the ray $R_{\bar{u}_{1}}=\left\{\left(u_{1}, v_{1}, u_{2}\right): u_{1}>\bar{u}_{1}\right.$ and $\left.v_{1}=u_{2}=0\right\} . H_{\bar{u}_{1}}$ is normally hyperbolic: the equilibria in $H_{\bar{u}_{1}}$ have two zero eigenvalues and one negative eigenvalue. (See Appendix A for an introduction to normally hyperbolic invariant manifolds.) $R_{\bar{u}_{1}}$ is also normally hyperbolic: the equilibria in $R_{\bar{u}_{1}}$ have one positive and one negative eigenvalue. To find a solution to the boundary value problem that satisfies the exponential approach condition, we need to find a solution in the intersection of $W^{u}\left(R_{\bar{u}_{1}}\right)$, the unstable manifold of the ray $R_{\bar{u}_{1}}$, which has dimension 2 , and $W^{s}(0,0,1)$, the stable manifold of the equilibrium $(0,0,1)$, which has dimension 1 . Of course these manifolds depend on the vector of parameters $\left(\beta, \bar{u}_{1}, \sigma\right)$.

The function $\left(u_{1}, v, u_{2}\right) \rightarrow \sigma u_{1}+v+\frac{\sigma}{\beta} u_{2}$ is a first integral of (3.6)-(3.8). To take advantage of this fact, we replace $v_{1}$ by $w_{1}$ defined by $w_{1}=\sigma u_{1}+v_{1}+\frac{\sigma}{\beta} u_{2}$. In the new variables, the differential equation (3.6)-(3.8) becomes

$$
\begin{aligned}
u_{1}^{\prime} & =-\sigma u_{1}+w_{1}-\frac{\sigma}{\beta} u_{2}, \\
w_{1}^{\prime} & =0, \\
u_{2}^{\prime} & =\frac{\beta}{\sigma} u_{2} \rho\left(u_{1}-\bar{u}_{1}\right) .
\end{aligned}
$$

Each plane $w_{1}=$ constant is invariant. Corresponding to $H_{\bar{u}_{1}}$ and $R_{\bar{u}_{1}}$ we have

$$
\begin{aligned}
& \tilde{H}_{\left(\beta, \bar{u}_{1}, \sigma\right)}=\left\{\left(u_{1}, w_{1}, u_{2}\right): u_{1} \leq \bar{u}_{1} \text { and } u_{2}=-\beta u_{1}+\frac{\beta}{\sigma} w_{1}\right\}, \\
& \tilde{R}_{\left(\beta, \bar{u}_{1}, \sigma\right)}=\left\{\left(u_{1}, w_{1}, u_{2}\right): u_{1}=\frac{1}{\sigma} w_{1}, w_{1}>\sigma \bar{u}_{1}, \text { and } u_{2}=0\right\} .
\end{aligned}
$$

For a fixed $w_{1}$ with $w_{1}>\sigma \bar{u}_{1}$, the phase portrait of (3.9), (3.11) is shown in Figure 3.1. The half-line of equilibria is part of $\tilde{H}_{\left(\beta, \bar{u}_{1}, \sigma\right)}$, and the isolated equilibrium on the $u_{1}$-axis is part of $\tilde{R}_{\left(\beta, \bar{u}_{1}, \sigma\right)}$. Where the unstable manifold of the isolated equilibrium goes depends on the vector of parameters $\left(\beta, \bar{u}_{1}, \sigma, w_{1}\right)$ in (3.9), (3.11).

Set $w_{1}=\frac{\sigma}{\beta}$, so that the equilibrium $\left(u_{1}, u_{2}\right)=\left(0, \frac{\beta}{\sigma} w_{1}\right)$ on the $u_{2}$ axis is at $(0,1)$ as desired. The isolated equilibrium is then $\left(u_{1}, u_{2}\right)=\left(\frac{1}{\beta}, 0\right)$, so we assume $\bar{u}_{1}<\frac{1}{\beta}$. If we now vary $\sigma$, it is not hard to see that for small $\sigma$, the unstable manifold of $\left(\frac{1}{\beta}, 0\right)$ lies above the stable manifold of $(0,1)$, and for large $\sigma$ it lies below [4,22]. Therefore there is a value $\sigma^{*}$ of $\sigma$ for which the unstable manifold of $\left(\frac{1}{\beta}, 0\right)$ meets the stable manifold of $(0,1)$. 


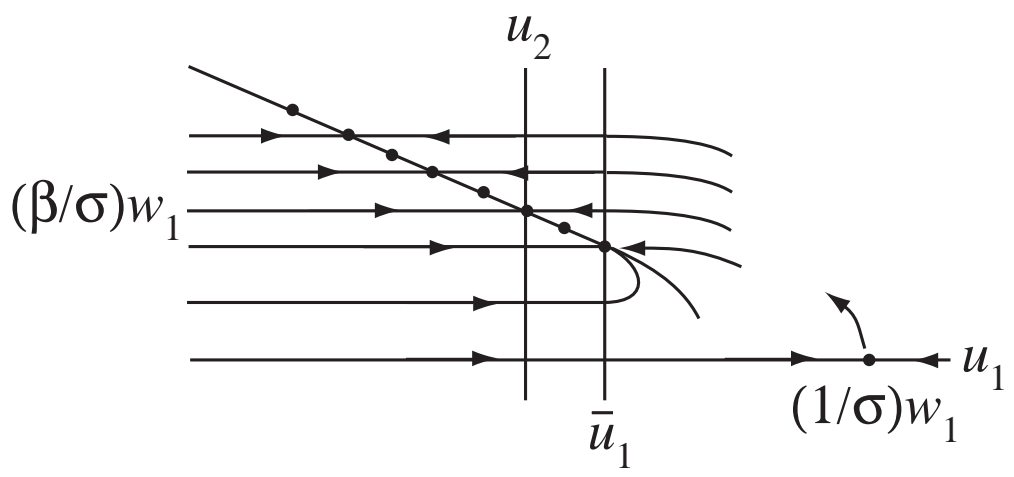

Figure 3.1. Phase portrait of (3.9), (3.11) for a fixed $w_{1}$ with $w_{1}>\sigma \bar{u}_{1}$.

For the system (3.9), (3.11), one can define a separation function $\tilde{S}_{0}\left(\beta, \bar{u}_{1}, \sigma\right)$ between the unstable manifold of $\left(\frac{1}{\beta}, 0\right)$ and the stable manifold of $(0,1) ; S_{0}$ is positive (respectively negative) when the former is above (respectively below) the latter. Then one can show that if $\tilde{S}_{0}\left(\beta, \bar{u}_{1}, \sigma\right)=0$ then $\frac{\partial \tilde{S}_{0}}{\partial \sigma}\left(\beta, \bar{u}_{1}, \sigma\right)<0$; this is done [8] by calculating an integral, called a Menikov integral, whose value gives the partial derivative. Therefore the unstable manifold of $\left(\frac{1}{\beta}, 0\right)$ always crosses from above the stable manifold of $(0,1)$ to below it as $\sigma$ increases. It follows that $\sigma^{*}$ is unique, and the intersection breaks in a nondegenerate manner as $\sigma$ varies. We let $\sigma^{*}=\sigma_{0}\left(\beta, \bar{u}_{1}\right)$; because the Melnikov integral is nonzero, $\sigma_{0}$ is smooth by the Implicit Function Theorem. For the given $\left(\beta, \bar{u}_{1}\right)$, the heteroclinic solution we have found is the unique traveling wave with positive velocity that satisfies the exponential approach condition, and $\sigma_{0}\left(\beta, \bar{u}_{1}\right)$ is its velocity.

$\tilde{S}_{0}\left(\beta, \bar{u}_{1}, \sigma\right)$ can be reinterpreted as referring to the system (3.9)-(3.11). It is then a separation function between the 2-dimensional manifold $W^{u}\left(\tilde{R}_{\left(\beta, \bar{u}_{1}, \sigma\right)}\right)$ and the 1-dimensional stable manifold of the equilibrium $\left(0, \frac{\sigma}{\beta}, 1\right)$.

Returning to $u_{1} v_{1} u_{2}$-space, for the system (3.6)-(3.8) we have a separation function $S_{0}\left(\beta, \bar{u}_{1}, \sigma\right)$ between the 2-dimensional manifold $W^{u}\left(R_{\bar{u}_{1}}\right)$ and the 1-dimensional stable manifold of the equilibrium $(0,0,1)$. When $S_{0}=0$, the two manifolds intersect in a heteroclinic solution from $\left(\frac{1}{\beta}, 0,0\right)$ to $(0,0,1)$. There is a smooth function $\sigma_{0}\left(\beta, \bar{u}_{1}\right)$ such that $S_{0}=0$ if and only if $\sigma=\sigma_{0}\left(\beta, \bar{u}_{1}\right)$, and $\frac{\partial S}{\partial \sigma}$ there is nonzero. In consequence we have

Theorem 3.1. For $\kappa=\delta=0$ and fixed $\left(\beta, \bar{u}_{1}\right)$, there is a unique speed $\sigma=\sigma_{0}\left(\beta, \bar{u}_{1}\right)$ such that the gasless combustion model (1.1)-(1.2) has a traveling wave of speed $\sigma$ that connects a burned state $\left(u_{1}^{*}, 0,0\right)$ to the unburned state $(0,0,1)$. The combustion temperature $u_{1}^{*}$ is $\frac{1}{\beta}$. The function $\sigma_{0}\left(\beta, \bar{u}_{1}\right)$ is smooth.

3.2. Infinite Lewis number $(\kappa=0)$ with heat loss $(\delta>0)$. We consider (3.3)-(3.4) with $\left(\beta, \bar{u}_{1}\right)$ fixed, $\delta>0$, and $\kappa=0$. We are interested in solutions with $\sigma>0$ that satisfy the boundary conditions

$$
\left(u_{1}, \partial_{\xi} u_{1}, u_{2}\right)(-\infty)=\left(0,0, u_{2}^{*}\right), \quad\left(u_{1}, \partial_{\xi} u_{1}, u_{2}\right)(\infty)=(0,0,1) .
$$

The unburned reactant concentration behind the front $u_{2}^{*}$ and the speed $\sigma$ are yet to be determined. 
As in the previous subsection, in the system (3.3)-(3.4) we set $v_{1}=\partial_{\xi} u_{1}$ and use prime to denote derivative with respect to $\xi$. We obtain the first-order system

$$
\begin{aligned}
u_{1}^{\prime} & =v_{1}, \\
v_{1}^{\prime} & =-\sigma v_{1}-u_{2} \rho\left(u_{1}-\bar{u}_{1}\right)+\delta u_{1}, \\
u_{2}^{\prime} & =\frac{\beta}{\sigma} u_{2} \rho\left(u_{1}-\bar{u}_{1}\right),
\end{aligned}
$$

which has the vector of parameters $\left(\beta, \bar{u}_{1}, \sigma, \delta\right)$. A solution of (3.3)-(3.4) that satisfies the boundary conditions (3.14) corresponds to a solution of (3.15)-(3.17) that goes from an equilibrium $\left(0,0, u_{2}^{*}\right)$ to the equilibrium $(0,0,1)$.

3.2.1. Equilibria. For $\delta>0$, the set of equilibria of (3.15)-(3.17) is the $u_{2}$-axis. The linearization of $(3.15)-(3.17)$ at a point $\left(u_{1}, v_{1}, u_{2}\right)$ has the matrix

$$
\left(\begin{array}{ccc}
0 & 1 & 0 \\
-u_{2} \rho^{\prime}\left(u_{1}-\bar{u}_{1}\right)+\delta & -\sigma & -\rho\left(u_{1}-\bar{u}_{1}\right) \\
\frac{\beta}{\sigma} u_{2} \rho^{\prime}\left(u_{1}-\bar{u}_{1}\right) & 0 & \frac{\beta}{\sigma} \rho\left(u_{1}-\bar{u}_{1}\right)
\end{array}\right)
$$

On the $u_{2}$-axis, (3.18) becomes

$$
\left(\begin{array}{ccc}
0 & 1 & 0 \\
\delta & -\sigma & 0 \\
0 & 0 & 0
\end{array}\right)
$$

so the equilibria all have the eigenvalues

$$
0 \quad \text { and } \quad \lambda_{ \pm}(\sigma, \delta)=-\frac{\sigma}{2} \pm\left(\frac{\sigma^{2}}{4}+\delta\right)^{\frac{1}{2}}
$$

Since $\delta>0$, we have $\lambda_{-}<0$ and $\lambda_{+}>0$. Therefore each equilibrium has a one-dimensional unstable manifold and a one-dimensional stable manifold. We need to find a solution that lies in the intersection of the unstable manifold of the $u_{2}$-axis, which is two-dimensional, and the stable manifold of $(0,0,1)$, which is one-dimensional.

For $\delta=0$ the equilibria are the half-plane $H_{\bar{u}_{1}}$ of the previous section, which we now denote $H_{\left(\beta, \bar{u}_{1}, \sigma, 0\right)}$ (the last component is $\delta$ ), and $R_{\bar{u}_{1}}$; see Figure 3.2 (a).

3.2.2. Normally hyperbolic invariant manifolds. For $\delta=0$, the unstable manifolds of points on $R_{\bar{u}_{1}}$ fit together with the half-plane $H_{\left(\beta, \bar{u}_{1}, \sigma, 0\right)}$ to make a normally attracting two-dimensional manifold that we denote $M_{\left(\beta, \bar{u}_{1}, \sigma, 0\right)}$. See Figure 3.2 (a). For an introduction to normally hyperbolic invariant manifolds, see Appendix A.

For small $\delta>0, M_{\left(\beta, \bar{u}_{1}, \sigma, 0\right)}$ perturbs to a two-dimensional normally attracting manifold $M_{\left(\beta, \bar{u}_{1}, \sigma, \delta\right)} . \quad M_{\left(\beta, \bar{u}_{1}, \sigma, \delta\right)}$ is just the $u_{2}$-axis, a line of equilibria, together with the unstable manifolds of these equilibria. One can check that $M_{\left(\beta, \bar{u}_{1}, \sigma, \delta\right)}$ includes the half-plane

$$
H_{\left(\beta, \bar{u}_{1}, \sigma, \delta\right)}=\left\{\left(u_{1}, v_{1}, u_{2}\right): u_{1} \leq \bar{u}_{1} \text { and } v_{1}=\lambda_{+}(\sigma, \delta) u_{1}\right\},
$$

and the line

$$
L_{\left(\beta, \bar{u}_{1}, \sigma, \delta\right)}=\left\{\left(u_{1}, v_{1}, u_{2}\right): v_{1}=\lambda_{+}(\sigma, \delta) u_{1} \text { and } u_{2}=0\right\} .
$$

(It is enough to check that these sets are invariant under (3.15)-(3.17) and near $M_{\left(\beta, \bar{u}_{1}, \sigma, 0\right)}$.) For $\delta>0$, the line $L_{\left(\beta, \bar{u}_{1}, \sigma, \delta\right)}$ is the unstable manifold of the origin. Note that $\lambda_{+}(\sigma, 0)=0$, so $L_{\left(\beta, \bar{u}_{1}, \sigma, 0\right)}$ contains $R_{u_{1}}$. See Figure $3.2(\mathrm{~b})$.

The system (3.15)-(3.17) restricted to $H_{\left(\beta, \bar{u}_{1}, \sigma, \delta\right)} \cup L_{\left(\beta, \bar{u}_{1}, \sigma, \delta\right)}$ is just $u_{1}^{\prime}=\lambda_{+}(\delta, \sigma) u_{1}, u_{2}^{\prime}=0$. 

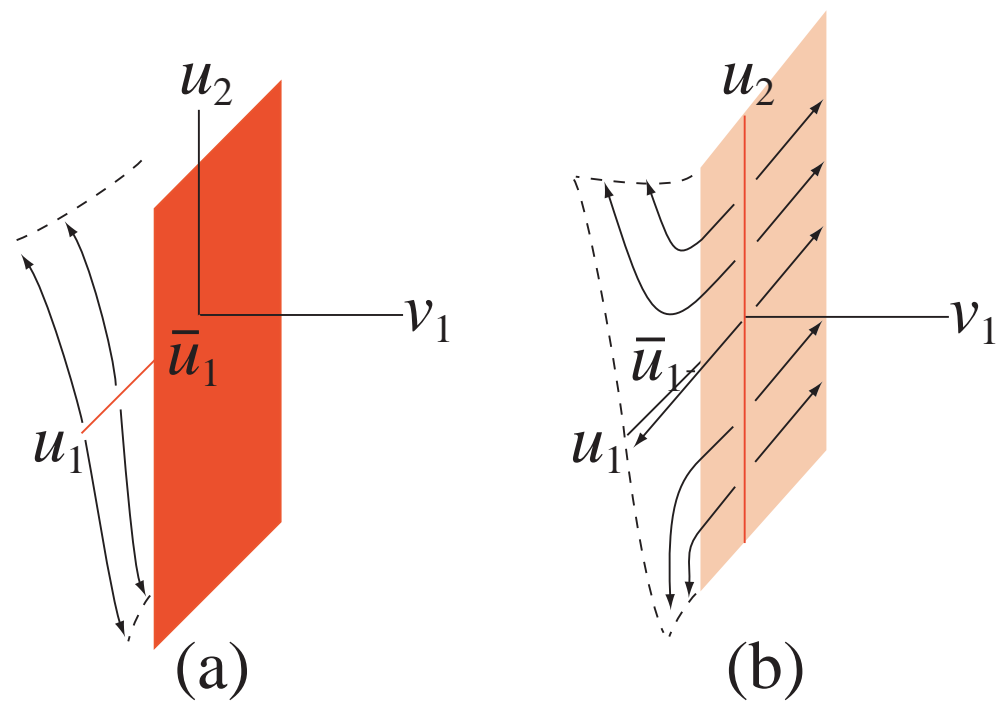

(b)

Figure 3.2. (a) The half-plane $H_{\left(\beta, \bar{u}_{1}, \sigma, 0\right)}$ of equilibria (red), and the ray of equilibria $R_{\bar{u}_{1}}$ (part of the $u_{1}$-axis, also red). The two-dimensional normally attracting manifold $M_{\left(\beta, \bar{u}_{1}, \sigma, 0\right)}$ for (3.6)-(3.8) is also shown; it includes both these sets, together with the unstable manifolds of points on $R_{\bar{u}_{1}}$. (b) The two-dimensional normally attracting manifold $M_{\left(\beta, \bar{u}_{1}, \sigma, \delta\right)}, \delta>0$, for (3.15)(3.17). It includes the $u_{2}$-axis, which is a line of equilibria, and the unstable manifolds of these equilibria. Within the half-plane $H_{\left(\beta, \bar{u}_{1}, \sigma, \delta\right)}$, which is part of $M_{\left(\beta, \bar{u}_{1}, \sigma, \delta\right)}$, the unstable manifolds are parallel lines. The entire unstable manifold of the origin is the line $L_{\left(\beta, \bar{u}_{1}, \sigma, \delta\right)}$, which lies in the plane $u_{2}=0$.

Theorem 3.2. For $\kappa=0$, fixed $\left(\beta, \bar{u}_{1}\right)$, and $\delta>0$ small, there is a unique speed $\sigma=$ $\sigma_{1}\left(\beta, \bar{u}_{1}, \delta\right)$ near the speed $\sigma_{0}\left(\beta, \bar{u}_{1}\right)$ of Theorem 3.1 such that the gasless combustion model (1.1)-(1.2) has a traveling wave of speed $\sigma$ that connects a burned state $\left(0,0, u_{2}^{*}\right)$ to the unburned state $(0,0,1)$. The unburned reactant concentration $u_{2}^{*}$ is positive, and $u_{2}^{*} \rightarrow 0$ as $\delta \rightarrow 0$. The function $\sigma_{1}\left(\beta, \bar{u}_{1}, \delta\right)$, defined for small $\delta \geq 0$, with $\sigma_{1}\left(\beta, \bar{u}_{1}, 0\right)=\sigma_{0}\left(\beta, \bar{u}_{1}\right)$, is smooth. As $\delta \rightarrow 0$, in $u_{1} v_{1} u_{2}$-space the corresponding heteroclinic solution of $(3.15)-(3.17)$ approaches the union of the heteroclinic solution with $\delta=0$ of Theorem 3.1, with speed $\sigma=\sigma_{0}\left(\beta, \bar{u}_{1}\right)$, and the line segment from $(0,0,0)$ to $\left(\frac{1}{\beta}, 0,0\right)$.

See Figure 3.3. The line segment from $(0,0,0)$ to $\left(\frac{1}{\beta}, 0,0\right)$ corresponds to declining temperature behind the combustion front due to heat loss to the environment.

To prove the theorem, just note that the separation function $S_{0}\left(\beta, \bar{u}_{1}, \sigma\right)$ of Subsection 3.1 extends to a separation function $S_{1}\left(\beta, \bar{u}_{1}, \sigma, \delta\right)$ between the two-dimensional manifold $M_{\left(\beta, \bar{u}_{1}, \sigma, \delta\right)}$ and the one-dimensional stable manifold of $(0,0,1)$; we have $S_{1}\left(\beta, \bar{u}_{1}, \sigma, 0\right)=$ $S_{0}\left(\beta, \bar{u}_{1}, \sigma\right)$. Since $S_{1}=0$ and $\frac{\partial S_{1}}{\partial \sigma} \neq 0$ at $\left(\beta, \bar{u}_{1}, \sigma_{0}\left(\beta, \bar{u}_{1}\right), 0\right.$, by the Implicit Function Theorem there is a function $\sigma_{1}\left(\beta, \bar{u}_{1}, \delta\right)$, with $\delta \geq 0$ small and $\sigma_{1}\left(\beta, \bar{u}_{1}, 0\right)=\sigma_{0}\left(\beta, \bar{u}_{1}\right)$, such that $S_{1}=0$ when $\sigma=\sigma_{1}\left(\beta, \bar{u}_{1}, \delta\right)$. For such $\sigma$, a branch of the stable manifold of $(0,0,1)$ lies in $M_{\left(\beta, \bar{u}_{1}, \sigma, \delta\right)}$. Since $M_{\left(\beta, \bar{u}_{1}, \sigma, \delta\right)}$ is foliated by the unstable manifolds of equilibria, in backward time it approaches an equilibrium $\left(0,0, u_{2}^{*}\right)$. We cannot have $u_{2}^{*}=0$ because our solution lies in the invariant set $u_{2}>0$, and the unstable manifold of the origin, $L_{\left(\beta, \bar{u}_{1}, \sigma, \delta\right)}$, lies in the 
invariant plane $u_{2}=0$. By continuity of the intersection of the invariant manifolds, $u_{2}^{*} \rightarrow 0$ as $\delta \rightarrow 0$.

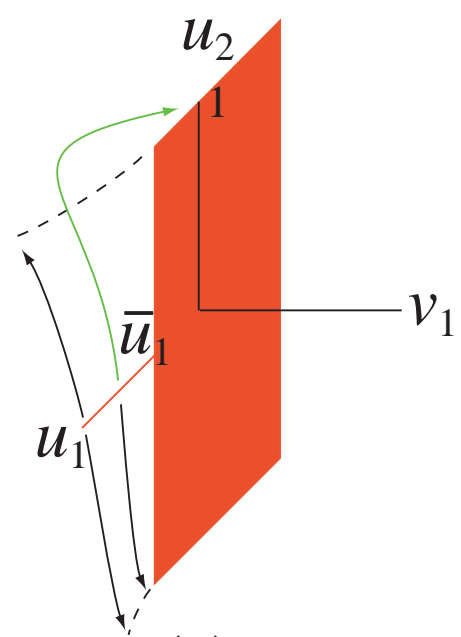

(a)

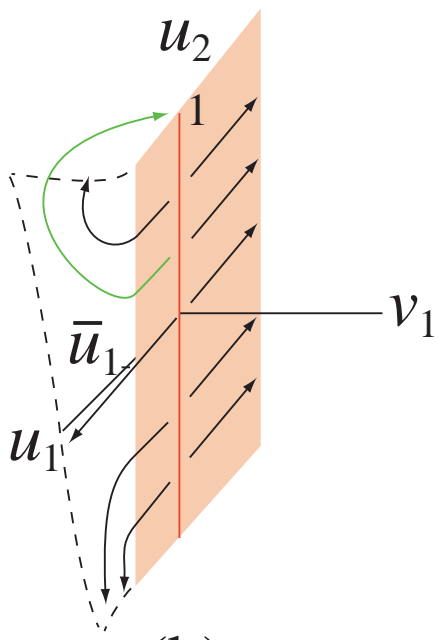

(b)

FIGURE 3.3. Invariant manifolds and connecting orbit for $\sigma=\sigma\left(\beta, \bar{u}_{1}, \delta\right)$ : (a) $\delta=0$ and $(\mathrm{b}) \delta>0$.

3.3. Finite Lewis number $(\kappa>0)$. The approach of this section was used in [2] and [7] for $\delta=0$.

We consider (3.3)-(3.4) with $\left(\beta, \bar{u}_{1}\right)$ fixed, $\delta \geq 0$, and $\kappa>0$. For $\delta=0$ we use the boundary conditions

$$
\left(u_{1}, \partial_{\xi} u_{1}, u_{2}, \partial_{\xi} u_{2}\right)(-\infty)=\left(u_{1}^{*}, 0,0,0\right), \quad\left(u_{1}, \partial_{\xi} u_{1}, u_{2}, \partial_{\xi} u_{2}\right)(\infty)=(0,0,1,0) .
$$

The combustion temperature $u_{1}^{*}$ and the speed $\sigma$ are yet to be determined. For $\delta>0$ we use the boundary conditions

$$
\left(u_{1}, \partial_{\xi} u_{1}, u_{2}, \partial_{\xi} u_{2}\right)(-\infty)=\left(0,0, u_{2}^{*}, 0\right), \quad\left(u_{1}, \partial_{\xi} u_{1}, u_{2}, \partial_{\xi} u_{2}\right)(\infty)=(0,0,1,0) .
$$

The unburned reactant concentration $u_{2}^{*}$ and the speed $\sigma$ are yet to be determined.

In the system (3.3)-(3.4) we set $v_{1}=\partial_{\xi} u_{1}, v_{2}=\partial_{\xi} u_{2}$, and use prime to denote derivative with respect to $\xi$. We obtain the first-order system

$$
\begin{aligned}
u_{1}^{\prime} & =v_{1}, \\
v_{1}^{\prime} & =-\sigma v_{1}-u_{2} \rho\left(u_{1}-\bar{u}_{1}\right)+\delta u_{1}, \\
u_{2}^{\prime} & =v_{2}, \\
\kappa v_{2}^{\prime} & =-\sigma v_{2}+\beta u_{2} \rho\left(u_{1}-\bar{u}_{1}\right) .
\end{aligned}
$$

This system has the vector of parameters $\left(\beta, \bar{u}_{1}, \sigma, \delta, \kappa\right)$. We restrict our attention to $\kappa>0$, $\delta \geq 0$, and $\sigma>0$. For $\kappa>0$, a solution of (3.3)-(3.4) that satisfies the boundary conditions (3.21) (respectively (3.22)) corresponds to a solution of (3.23)-(3.26) that goes from an equilibrium $\left(u_{1}^{*}, 0,0,0\right)$ (respectively $\left.\left(0,0, u_{2}^{*}, 0\right)\right)$ to, in both cases, the equilibrium $(0,0,1,0)$.

For $\kappa>0$ and $\delta=0$, the set of equilibria of (3.23)-(3.26) is the half plane $\hat{H}_{\bar{u}_{1}}=$ $\left\{\left(u_{1}, v_{1}, u_{2}, v_{2}\right): u_{1} \leq \bar{u}_{1}\right.$ and $\left.v_{1}=v_{2}=0\right\}$, together with the ray $\hat{R}_{\bar{u}_{1}}=\left\{\left(u_{1}, v_{1}, u_{2}, v_{2}\right)\right.$ : 
$u_{1}>\bar{u}_{1}$ and $\left.v_{1}=u_{2}=v_{2}=0\right\} . \quad H_{\bar{u}_{1}}$ is normally attracting: the equilibria in $\hat{H}_{\bar{u}_{1}}$ have two negative eigenvalues. $\hat{R}_{\bar{u}_{1}}$ is also normally hyperbolic: the equilibria in $\hat{R}_{\bar{u}_{1}}$ have one positive and two negative eigenvalue. To solve the boundary value problem (3.23)-(3.26), (3.21), we need to find a solution in the intersection of $W^{u}\left(\hat{R}_{\bar{u}_{1}}\right)$, which has dimension 2, and $\hat{W}^{s}(0,0,1,0)$, which also has dimension 2 .

For $\kappa>0$ and $\delta>0$, the set of equilibria of (3.23)-(3.26) is the $u_{2}$-axis. Linearization shows that each equilibrium has a one-dimensional unstable manifold and a two-dimensional stable manifold. To solve the boundary value problem (3.23)-(3.26), (3.22), we need to find a solution in the intersection of the unstable manifold of the $u_{2}$-axis, which has dimension 2 , and $W^{s}(0,0,1)$, which also has dimension 2 .

By rescaling time, the system (3.23)-(3.26) can be converted to

$$
\begin{aligned}
& \dot{u}_{1}=\kappa v_{1}, \\
& \dot{v}_{1}=\kappa\left(-\sigma v_{1}-u_{2} \rho\left(u_{1}-\bar{u}_{1}\right)+\delta u_{1}\right), \\
& \dot{u}_{2}=\kappa v_{2}, \\
& \dot{v}_{2}=-\sigma v_{2}+\beta u_{2} \rho\left(u_{1}-\bar{u}_{1}\right) .
\end{aligned}
$$

In geometric singular perturbation theory, with $\kappa$ regarded as small, (3.23)-(3.26) is the slow form of a slow-fast system; (3.27)-(3.30) is the fast form.

For $\kappa=0$, the set of equilibria of (3.27)-(3.30) is the 3-dimensional manifold (slow manifold)

$$
P_{\left(\beta, \bar{u}_{1}, \sigma, \delta, 0\right)}=\left(\left\{\left(u_{1}, v_{1}, u_{2}, v_{2}\right): v_{2}=\frac{\beta}{\sigma} u_{2} \rho\left(u_{1}-\bar{u}_{1}\right)\right\} .\right.
$$

These equilibria have the negative eigenvalue $-\sigma$, so the slow manifold is normally attracting.

For small $\kappa>0, P_{\left(\beta, \bar{u}_{1}, \sigma, \delta, 0\right)}$ perturbs to a normally attracting manifold $P_{\left(\beta, \bar{u}_{1}, \sigma, \delta, \kappa\right)}$. These manifolds are parameterized by $\left(u_{1}, v_{1}, u_{2}\right)$. In terms of these variables, the system (3.27)(3.30), restricted to $P_{\left(\beta, \bar{u}_{1}, \sigma, \delta, \kappa\right)}$, is given to lowest order by (3.27)-(3.29) with $v_{2}=\frac{\beta}{\sigma} u_{2} \rho\left(u_{1}-\right.$ $\left.\bar{u}_{1}\right)$. After division by $\kappa$ (undoing the rescaling that produced (3.27)-(3.30)), we have (3.15)(3.17). In geometric singular perturbation theory, this system is called the slow system on the slow manifold.

The 2-dimensional manifold $M_{\left(\beta, \bar{u}_{1}, \sigma, \delta\right)}$ of the previous subsection, and the one-dimensional stable manifold of $(0,0,1)$ used in that subsection, correspond to submanifolds of $P_{\left(\beta, \bar{u}_{1}, \sigma, \delta, 0\right)}$ that we denote $M_{\left(\beta, \bar{u}_{1}, \sigma, \delta, 0\right)}$ and $N_{\left(\beta, \bar{u}_{1}, \sigma, \delta, 0\right)}$.

For small $\kappa>0$, these manifolds perturb to invariant manifolds $M_{\left(\beta, \bar{u}_{1}, \sigma, \delta, \kappa\right)}$ and $N_{\left(\beta, \bar{u}_{1}, \sigma, \delta, \kappa\right)}$ of $P_{\left(\beta, \bar{u}_{1}, \sigma, \delta, \kappa\right)} \cdot M_{\left(\beta, \bar{u}_{1}, \sigma, \delta, \kappa\right)}$ contains:

- for $\delta=0$, the 2-dimensional unstable manifold of the ray $\hat{R}_{\bar{u}_{1}}$ of equilibria;

- for $\delta>0$, the 2-dimensional unstable manifold of the $u_{2}$-axis.

$N\left(\beta, \bar{u}_{1}, \sigma, \delta, \kappa\right)$ is a 1 -dimensional portion of the 2 -dimensional stable manifold of $(0,0,0,1)$.

The separation function $S_{1}\left(\beta, \bar{u}_{1}, \sigma, \delta\right)$ used in the proof of Theorem 3.2 extends to a separation function $S\left(\beta, \bar{u}_{1}, \sigma, \delta, \kappa\right)$ between $M_{\left(\beta, \bar{u}_{1}, \sigma, \delta, \kappa\right)}$ and $N_{\left(\beta, \bar{u}_{1}, \sigma, \delta, \kappa\right)}$; we have $S\left(\beta, \bar{u}_{1}, \sigma, \delta, 0\right)=$ $S_{1}\left(\beta, \bar{u}_{1}, \sigma, \delta\right)$. At points $\left(\beta, \bar{u}_{1}, \sigma, \delta, \kappa\right)$ with $\sigma=\sigma_{1}\left(\beta, \bar{u}_{1}, \delta\right)$ and $\kappa=0$, we have $S=0$ and the partial derivative of $\frac{\partial S}{\partial \sigma} \neq 0$. Therefore, by the Implicit Function Theorem, there is a smooth function $\sigma\left(\beta, \bar{u}_{1}, \delta, \kappa\right)$, with $\kappa \geq 0$ small and $\sigma\left(\beta, \bar{u}_{1}, \delta, 0\right)=\sigma_{1}\left(\beta, \bar{u}_{1}, \delta\right)$, such that $S=0$ when $\sigma=\sigma\left(\beta, \bar{u}_{1}, \delta, \kappa\right)$. For such $\sigma, N_{\left(\beta, \bar{u}_{1}, \sigma, \delta, \kappa\right)}$ lies in $M_{\left(\beta, \bar{u}_{1}, \sigma, \delta, \kappa\right)}$.

For $\delta>0, \kappa>0$, and such $\sigma$, in backward time $N_{\left(\beta, \bar{u}_{1}, \sigma, \delta, \kappa\right)}$ approaches an equilibrium, since $M_{\left(\beta, \bar{u}_{1}, \sigma, \delta, \kappa\right)}$ is foliated by the unstable manifolds of equilibria. That equilibrium cannot 
be the origin because it is easy to see that the unstable manifold of the origin lies in the invariant set $u_{2}=v_{2}=0$.

We have proved:

Theorem 3.3. For fixed $\left(\beta, \bar{u}_{1}\right)$, and small $\delta \geq 0$ and $\kappa \geq 0$, there is a speed $\sigma=$ $\sigma\left(\beta, \bar{u}_{1}, \delta, \kappa\right)$, with $\sigma\left(\beta, \bar{u}_{1}, \delta, 0\right)=\sigma_{1}\left(\beta, \bar{u}_{1}, \delta\right)$, such that the gasless combustion model (1.1)(1.2) has a traveling wave of speed $\sigma$ with the following properties:

(1) For $\kappa>0$ and $\delta=0$, the wave connects a burned state $\left(u_{1}^{*}, 0,0,0\right)$ to the unburned state $(0,0,1,0)$. The combustion temperature $u_{1}^{*}$ is positive, and $u_{1}^{*} \rightarrow \frac{1}{\beta}$ as $\delta \rightarrow 0$.

(2) For $\kappa>0$ and $\delta>0$, the wave connects a burned state $\left(0,0, u_{2}^{*}, 0\right)$ to the equilibrium $(0,0,1,0)$. The unburned reactant concentration $u_{2}^{*}$ is positive, and $u_{2}^{*} \rightarrow 0$ as $\delta \rightarrow 0$.

The function $\sigma\left(\beta, \bar{u}_{1}, \delta, \kappa\right)$ is smooth. For small $\delta \geq 0$ and $\kappa \geq 0$, there are no other traveling waves with speed near $\sigma_{0}\left(\beta, \bar{u}_{1}\right)$.

Remark 3.4. There is considerable numerical evidence, such as Figure 2.1, showing that for $\delta$ positive and small, there is a traveling wave with speed near zero. So far as we know, there is no theoretical analysis of the asymptotic behavior of this wave as $\delta \rightarrow 0$ with $\left(\beta, \bar{u}_{1}, \kappa\right)$ fixed.

\section{Stability of the traveling WAVES}

For $\delta=0$, we refer to [8] for $\kappa=0$ and to [6] for $\kappa>0$, although the proof given below would work for $\delta=0$ as well with small changes.

Theorems 3.14 and 3.16 of [10] allow one to conclude the stability results sketched in the introduction, once one has checked that the nonlinear term has the correct form and verified some spectral conditions in various spaces. In this section we do the verifications and state the conclusions. The weighted spaces that are used depend on the rate of approach of the traveling wave to its end states, so we check that as well.

Fix $\left(\beta, \bar{u}_{1}, \delta, \kappa\right)$ with $\delta>0$. Let $\left(u_{1 *}, u_{2 *}\right)(\xi)$ be a solution of (3.3)-(3.4), with $\sigma=$ $\sigma\left(\beta, \bar{u}_{1}, \delta, \kappa\right)$, and with boundary values given by (3.14) when $\kappa=0$ and by (3.22) when $\kappa>0$.

In order to study the stability of this traveling wave using the theory of [10], we must first shift its left state to the origin.

To do this, let $z_{2}=u_{2}-u_{2}^{*}$. The PDE system (3.1)-(3.2) becomes

$$
\begin{aligned}
& \partial_{t} u_{1}=\partial_{\xi \xi} u_{1}+\sigma \partial_{\xi} u_{1}+\left(u_{2}^{*}+z_{2}\right) \rho\left(u_{1}-\bar{u}_{1}\right)-\delta u_{1}, \\
& \partial_{t} z_{2}=\kappa \partial_{\xi \xi} z_{2}+\sigma \partial_{\xi} z_{2}-\beta\left(u_{2}^{*}+z_{2}\right) \rho\left(u_{1}-\bar{u}_{1}\right) .
\end{aligned}
$$

We write (4.1)-(4.2) as $Y_{t}=D Y_{\xi \xi}+\sigma Y_{\xi}+R(Y)$ with

$$
Y=\left(\begin{array}{l}
u_{1} \\
z_{2}
\end{array}\right), \quad D=\left(\begin{array}{cc}
1 & 0 \\
0 & \kappa
\end{array}\right), \quad R\left(\begin{array}{l}
u_{1} \\
z_{2}
\end{array}\right)=\left(\begin{array}{c}
\left(u_{2}^{*}+z_{2}\right) \rho\left(u_{1}-\bar{u}_{1}\right)-\delta u_{1} \\
-\beta\left(u_{2}^{*}+z_{2}\right) \rho\left(u_{1}-\bar{u}_{1}\right)
\end{array}\right) .
$$

Note that $R\left(0, z_{2}\right) \equiv 0$ but $R\left(u_{1}, 0\right)$ is not identically 0 . One of these conditions is needed to use the results of [10].

Let $z_{2 *}(\xi)=u_{2 *}(\xi)-u_{2}^{*}$. Then $Y_{*}(\xi)=\left(u_{1 *}, z_{2 *}\right)(\xi)$ is an equilibrium solution of $(4.1)-$ (4.2), with $Y_{*}(-\infty)=Y_{-}=(0,0)$ and $Y_{*}(\infty)=Y_{+}=\left(0,1-u_{2}^{*}\right)$. 
4.1. Rate of approach of $Y_{*}(\xi)$ to end states. Recall the numbers $\lambda_{ \pm}(\sigma, \delta)$ given by (3.20). For $\kappa=0,(3.20)$ implies that there is a number $K>0$ such that

$$
\text { for } \xi \leq 0,\left\|Y_{*}(\xi)\right\| \leq K e^{\lambda_{+}(\sigma, \delta) \xi},
$$

and

$$
\text { for } \xi \geq 0,\left\|Y_{*}(\xi)-Y_{+}\right\| \leq K e^{\lambda_{-}(\sigma, \delta) \xi} .
$$

In the case $\kappa>0$, the traveling wave equation is (3.23)-(3.26), and the eigenvalues of the linearization at any equilibrium are $\lambda_{ \pm}(\sigma, \delta), 0$, and $-\frac{\sigma}{\kappa}$. For small $\kappa>0$,

$$
-\frac{\sigma}{\kappa}<\lambda_{-}(\sigma, \delta)<0<\lambda_{+}(\sigma, \delta)
$$

Therefore for small $\kappa>0$ we have (4.3), and, because the traveling waves constructed in Subsection 3.3 lie in the normally attracting invariant manifold of that subsection, we have (4.4).

4.2. Linearization. The linearization of $(4.1)-(4.2)$ at $Y_{*}(\xi)$ is

$$
\tilde{Y}_{t}=L \tilde{Y}=D \tilde{Y}_{\xi \xi}+\sigma \tilde{Y}_{\xi}+D R\left(Y_{*}(\xi)\right) \tilde{Y} .
$$

Note that $D R\left(Y_{ \pm}\right) \tilde{Y}=\operatorname{diag}(-\delta, 0) \tilde{Y}$, so the linearization of (4.1)-(4.2) at the constant solutions $Y_{ \pm}$is just

$$
\tilde{Y}_{t}=L^{ \pm} \tilde{Y}=D \tilde{Y}_{\xi \xi}+\sigma \tilde{Y}_{\xi}+\operatorname{diag}(-\delta, 0) \tilde{Y}
$$

Thus $L^{-}=L^{+}$.

We shall refer to " $L$ on $L^{2}$ " when we should more properly say "the operator defined by $L$ on $L^{2}(\mathbb{R})$, with its natural domain," etc.

The spectrum of $L^{ \pm}$on $L^{2}$ (and hence on $H^{1}$ or on $B U C$, the space of bounded uniformly continuous functions with the sup norm) can be computed by Fourier transform. It is the union of two curves, $\left\{\lambda=\theta+i \omega: \theta=-\left(\frac{\omega}{\sigma}\right)^{2}-\delta\right\}$, which is the spectrum of $\partial_{\xi \xi}+\sigma \partial_{\xi}-\delta$, and $\left\{\lambda=\theta+i \omega: \theta=-\kappa\left(\frac{\omega}{\sigma}\right)^{2}\right\}$, which is the spectrum of $\kappa \partial_{\xi \xi}+\sigma \partial_{\xi}$. The right-hand boundary of this set of two curves is the right-hand boundary of the essential spectrum of $L$ on $L^{2}$ or $H^{1}$ or $B U C$.

4.3. Weighted spaces. Given $\alpha=\left(\alpha_{-}, \alpha_{+}\right) \in \mathbb{R}^{2}$, let $\gamma_{\alpha}(\xi)$ be a smooth positive function that equals $e^{\alpha_{-} \xi}$ for $\xi \leq-1$ and equals $e^{\alpha_{+} \xi}$ for $\xi \geq 1$. As in the Introduction, let $\mathcal{E}_{0}$ denote $H^{1}$ or $B U C$, with norm (the unweighted norm) \|\|$_{0}$. Let $\mathcal{E}_{\alpha}$ denote the corresponding weighted space with weight function $\gamma_{\alpha}: u \in \mathcal{E}_{\alpha}$ if and only if $\gamma_{\alpha}(\xi) u(\xi) \in \mathcal{E}_{0}$, and $\|u\|_{\alpha}=\left\|\gamma_{\alpha}(\xi) u(\xi)\right\|_{0}$. (This definition of $\mathcal{E}_{\alpha}$ is a little more general than that used in the Introduction.)

The previous subsection shows that on $\mathcal{E}_{0}$ the essential spectrum of $L$ touches the imaginary axis. On the other hand, we have the following result, which is needed to use the theory of $[10]$.

Lemma 4.1. Consider the system (4.1)-(4.2) with $\delta>0$ and $\kappa \geq 0$ small, and $\sigma=$ $\sigma\left(\beta, \bar{u}_{1}, \delta, \kappa\right)$. Suppose $0<\alpha_{-}<\lambda_{+}(\sigma, \delta)$ and $0<\alpha_{+}<-\lambda_{-}(\sigma, \delta)$. Then the essential spectrum of $L$ on $\mathcal{E}_{\alpha}$ is contained in a half-plane $\operatorname{Re} \lambda \leq-\nu<0$.

The proof of the lemma is a calculation. The operator $L$ on $\mathcal{E}_{\alpha}$ is similar to the operator $\gamma_{\alpha} L \gamma_{\alpha}^{-1}$ on $\mathcal{E}_{0}$, and hence has the same spectrum. The latter is given by

$$
\hat{L} W=\gamma_{\alpha} L \gamma_{\alpha}^{-1} W
$$


Setting $\xi= \pm \infty$ in (4.6) yields

$$
\hat{L}^{ \pm} W=\left(\begin{array}{cc}
\partial_{\xi \xi}+\left(\sigma-2 \alpha_{ \pm}\right) \partial_{\xi}+\left(\alpha_{ \pm}^{2}-\sigma \alpha_{ \pm}-\delta\right) & 0 \\
0 & \kappa \partial_{\xi \xi}+\left(\sigma-2 \kappa \alpha_{ \pm}\right) \partial_{\xi}+\left(\kappa \alpha_{ \pm}^{2}-\sigma \alpha_{ \pm}\right.
\end{array}\right) W .
$$

The spectrum of $\hat{L}^{-}$on $\mathcal{E}_{0}$ is the union of two curves,

$$
\left\{\lambda=\theta+i \omega: \theta=-\left(\frac{\omega}{\sigma-2 \alpha_{-}}\right)^{2}+\left(\alpha_{-}^{2}-\sigma \alpha_{-}-\delta\right)\right\},
$$

which is the spectrum of $\partial_{\xi \xi}+\left(\sigma-2 \alpha_{-}\right) \partial_{\xi}+\left(\alpha_{-}^{2}-\sigma \alpha_{-}-\delta\right)$, and

$$
\left\{\lambda=\theta+i \omega: \theta=-\kappa\left(\frac{\omega}{\sigma-2 \alpha_{-}}\right)^{2}+\left(\kappa \alpha_{-}^{2}-\sigma \alpha_{-}\right)\right\},
$$

which is the spectrum of $\kappa \partial_{\xi \xi}+\left(\sigma-2 \kappa \alpha_{-}\right) \partial_{\xi}+\left(\kappa \alpha_{-}^{2}-\sigma \alpha_{-}\right)$. An analogous result holds for the spectrum of $\hat{L}^{+}$on $\mathcal{E}_{0}$.

From the choice of $\alpha_{ \pm}$in the lemma, we have

$$
\begin{gathered}
0<\alpha_{-}<\lambda_{+}(\sigma, \delta)=-\frac{\sigma}{2}+\left(\frac{\sigma^{2}}{4}+\delta\right)^{\frac{1}{2}}, \\
0<\alpha_{+}<-\lambda_{-}(\sigma, \delta)=\frac{\sigma}{2}+\left(\frac{\sigma^{2}}{4}+\delta\right)^{\frac{1}{2}} .
\end{gathered}
$$

If $\kappa>0,(4.5)$ implies

$$
\alpha_{-}<\lambda_{+}(\sigma, \delta)<-\lambda_{-}(\sigma, \delta)<\frac{\sigma}{\kappa} \quad \text { and } \quad \alpha_{+}<-\lambda_{-}(\sigma, \delta)<\frac{\sigma}{\kappa} .
$$

Therefore $\kappa \alpha_{-}^{2}-\sigma \alpha_{-}<0$ and $\kappa \alpha_{+}^{2}-\sigma \alpha_{-}<0$. Of course these statements are also true when $\kappa=0$. Therefore:

(1) The spectrum of $\hat{L}^{-}$on $\mathcal{E}_{0}$ lies in $\operatorname{Re} \lambda \leq-\nu_{-}=\max \left(\alpha_{-}^{2}-\sigma \alpha_{-}-\delta, \kappa \alpha_{-}^{2}-\sigma \alpha_{-}\right)<0$.

(2) The spectrum of $\hat{L}^{+}$on $\mathcal{E}_{0}$ lies in $\operatorname{Re} \lambda \leq-\nu_{+}=\max \left(\alpha_{+}^{2}-\sigma \alpha_{+}-\delta, \kappa \alpha_{+}^{2}-\sigma \alpha_{+}\right)<0$. The lemma follows.

4.4. Results. Our stability result is:

Theorem 4.2. Consider the system (4.1)-(4.2) with $\delta>0$ and $\kappa \geq 0$ small, and $\sigma=$ $\sigma\left(\beta, \bar{u}_{1}, \delta, \kappa\right)$. Let $\alpha_{-}$and $\alpha_{+}$be chosen as in Lemma 4.1. Let $\alpha=\left(\alpha_{-}, \alpha_{+}\right)$and let $\eta=$ $\left(0, \alpha_{+}\right)$. Assume in addition that the Evans function for the traveling wave $Y_{*}(\xi)$ has no zeros in the half-plane $\operatorname{Re} \lambda \geq 0$ other than a simple zero at the origin. Suppose $Y^{0} \in Y_{*}+\mathcal{E}_{\eta}^{2}$ with $\left\|Y^{0}-Y_{*}\right\|_{\eta}$ small, and let $Y(t)$ be the solution of $(4.1)-(4.2)$ in $Y_{*}+\mathcal{E}_{\eta}^{2}$ with $Y(0)=Y^{0}$. Then:

(1) $Y(t)$ is defined for all $t \geq 0$.

(2) $Y(t)=\tilde{Y}(t)+Y_{*}(\xi-q(t))$ with $\tilde{Y}(t)$ in a fixed subspace of $\mathcal{E}_{\eta}^{2}$ complementary to the span of $Y_{*}^{\prime}$. Let $\tilde{Y}(t)=\left(\tilde{u}_{1}(t), \tilde{z}_{2}(t)\right)$.

(3) $\|\tilde{Y}(t)\|_{\eta}+|q(t)|$ is small for all $t \geq 0$.

(4) $\|\tilde{Y}(t)\|_{\alpha}$ decays exponentially as $t \rightarrow \infty$.

(5) There exists $q^{*}$ such that $\left|q(t)-q^{*}\right|$ decays exponentially as $t \rightarrow \infty$.

(6) There is a constant $C$ independent of $Y^{0}$ such that $\left\|\tilde{z}_{2}(t)\right\|_{0} \leq C\left\|\tilde{Y}^{0}\right\|_{\eta}$ for all $t \geq 0$.

(7) $\left\|\tilde{u}_{1}(t)\right\|_{0}$ decays exponentially as $t \rightarrow \infty$. 
This theorem follows from Theorem 3.14 in [10]. The hypotheses of that theorem are verified by Lemma 4.1 and the following observations:

(1) $R\left(0, z_{2}\right) \equiv 0$ (noted at the end of Subsection 4.1).

(2) The operator $\kappa \partial_{\xi \xi}+\sigma \partial_{\xi}$ on $\mathcal{E}_{0}$ generates a bounded semigroup.

(3) The operator $\partial_{\xi \xi}+\sigma \partial_{\xi}-\delta$ on $\mathcal{E}_{0}$ has its spectrum contained in $\operatorname{Re} \lambda \leq-\delta<0$.

We remark that for a fixed $\delta>0$, if the Evans function condition in Theorem 4.2 holds for $\kappa=0$, then it holds for small $\kappa>0$. The analogous fact for $\delta=0$ is proved in [7]. The proof for $\delta>0$ is similar but easier.

Theorem 3.16 in [10] implies some additional conclusions when $\kappa>0$ in Theorem 4.2. Consider the Banach space $\mathcal{E}_{0} \cap L^{1}(\mathbb{R})$ with the norm $\|u\|_{\mathcal{E}_{0} \cap L^{1}(\mathbb{R})}=\max \left\{\|u\|_{\mathcal{E}_{0}},\|u\|_{L^{1}(\mathbb{R})}\right\}$. Suppose $Y^{0} \in Y_{*}+\left(\mathcal{E}_{\eta} \cap L^{1}(\mathbb{R})\right)^{2}$ with $\left\|Y^{0}-Y_{*}\right\|_{\eta}$ and $\left\|Y^{0}-Y_{*}\right\|_{L^{1}}$ sufficiently small, and as in Theorem 4.2 let $Y(t)$ be the solution of $(4.1)-(4.2)$ in in $Y_{*}+\mathcal{E}_{\eta}^{2}$ with $Y(0)=Y^{0}$. Let $h(t)=\min \left(1, t^{-\frac{1}{2}}\right)$. Then for fixed $\kappa>0$ and for all $t \geq 0$ :

(1) $Y(t) \in\left(\mathcal{E}_{\eta} \cap L^{1}(\mathbb{R})\right)^{2}$.

(2) There is a constant $C$ independent of $Y^{0}$ such that

$$
\left.\left\|\tilde{z}_{2}(t)\right\|_{L^{1}} \leq C \max \left(\| \tilde{z}_{2}^{0}\right)\left\|_{L^{1}},\right\| \tilde{Y}^{0} \|_{\alpha}\right) \quad \text { and } \quad\left\|\tilde{z}_{2}\right\|_{L^{\infty}} \leq C h(t) \max \left(\left\|\tilde{z}_{2}^{0}\right\|_{L^{1}},\left\|\tilde{Y}^{0}\right\|_{\eta}\right) .
$$

(3) $\left\|\tilde{u}_{1}\right\|_{L^{1}}$ decays exponentially as $t \rightarrow \infty$.

\section{EVANS FUNCTION}

We continue to assume that $\delta>0$ and $\kappa \geq 0$ are small, so that (4.4) and (4.5) hold, and that $\alpha_{-}$and $\alpha_{+}$are chosen as in Lemma 4.1.

5.1. Definition. For fixed $\left(\beta, \bar{u}_{1}, \delta, \kappa\right)$ with $\kappa>0$, we write the traveling wave system (3.23)(3.26) as $X_{\xi}=F(X, \sigma)$. Let $X_{*}(\xi)=\left(u_{1 *}, u_{1 *}^{\prime}, u_{2 *}, u_{2 *}^{\prime}\right)(\xi)$, with velocity $\sigma_{*}=\sigma\left(\beta, \bar{u}_{1}, \delta, \kappa\right)$, and let $B(\xi)=F_{X}\left(X_{*}(\xi), \sigma_{*}\right)$. (The prime denotes derivative with respect to $\xi$.)

The complex number $\lambda$ is an eigenvalue of $L$ on $\mathcal{E}_{\alpha}$ with eigenfunction $\tilde{Y}$ provided $\tilde{Y} \in \mathcal{E}_{\alpha}^{2}$ and $\lambda \tilde{Y}=L \tilde{Y}$. This second-order linear differential equation can be written as the first-order system on $\mathbb{R}^{4}$

$$
X_{\xi}=(B(\xi)+\lambda C) X
$$

with $C$ a constant matrix. If $X$ is a solution of (5.1) in $\mathcal{E}_{\alpha}^{4}$, then the first and third components of $X$ give a solution $\tilde{Y}$ of $\tilde{Y}_{t}=L \tilde{Y}$ in $\mathcal{E}_{\alpha}^{2}$; and every such solution of $\tilde{Y}_{t}=L \tilde{Y}$ arises this way.

Recall the numbers $-\nu_{ \pm}<0$ defined in the proof of Lemma 4.1. For $\operatorname{Re} \lambda \geq-\frac{1}{2} \nu_{-},(5.1)$ has linear independent solutions $X^{1}(\xi, \lambda)$ and $X^{2}(\xi, \lambda)$ such that $e^{\alpha_{-} \xi} X^{i}(\xi), i=1$, 2, is bounded for $\xi \leq 0$. (For $\operatorname{Re} \lambda>0$ these two solutions decay at the left; to extend them to $\operatorname{Re} \lambda \geq-\frac{1}{2} \nu_{-}$we must allow them to grow slowly at the left.) Similarly, for $\operatorname{Re} \lambda \geq-\frac{1}{2} \nu_{+}$, (5.1) has linear independent solutions $X^{3}(\xi, \lambda)$ and $X^{4}(\xi, \lambda)$ such that $e^{\alpha+\xi} X^{i}(\xi, \lambda), i=3,4$, is bounded for $\xi \geq 0$. By a theorem of Kato [14], these solutions may be chosen to depend analytically on $\lambda$. The Evans function $D(\lambda)$ is defined for $\operatorname{Re} \lambda \geq \max \left(-\frac{1}{2} \nu_{-},-\frac{1}{2} \nu_{+}\right)$by

$$
D(\lambda)=\operatorname{det}\left(X^{1}(0, \lambda), \ldots, X^{4}(0, \lambda)\right) .
$$

It is 0 if and only if $\lambda$ is an eigenvalue of $L$ on $\mathcal{E}_{\alpha}$. 
5.2. Derivative at $\lambda=0$. Two general facts about the Evans function are (1) $D(0)=0$ because the derivative of the traveling wave is an eigenfunction of $L$ for the eigenvalue 0 , and (2) nonzero derivative of the separation function used to construct the traveling wave implies $\frac{d D}{d \lambda}(0) \neq 0$. In this subsection we sketch the argument for the present situation.

For $\delta \geq 0$ and $\kappa>0$ small, along $X_{*}(\xi)$, the two-dimensional tangent spaces to $M_{\left(\beta, \bar{u}_{1}, \sigma^{*}, \delta, \kappa\right)}$ (defined in Subsection 3.3) and to the stable manifold of $(0,0,1,0)$ have just a one-dimensional intersection. Then the $X^{i}(\xi, 0)$ span a space of dimension 3, and they can be chosen so that $X^{1}(\xi, 0)=X^{3}(\xi, 0)=X_{*}^{\prime}(\xi)$, which we assume. Hence up to scalar multiplication there is a unique solution $\psi_{*}(\xi)$ in $\mathcal{E}_{\left(-\alpha_{+},-\alpha_{-}\right)}^{4}$ of the adjoint equation $\psi_{\xi}=-B(\xi)^{\top} \psi$. We have $\psi_{*}(\xi)^{\top} X^{i}(\xi, 0)=0$ for $i=1, \ldots, 4$, and, according to [19], up to a nonzero multiple,

$$
\frac{d D}{d \lambda}(0)=\int_{-\infty}^{\infty} \psi_{*}(\xi)^{\top} C X_{*}^{\prime}(\xi) d \xi
$$

Let us further assume that the derivative of the separation function used in subsection 3.3 with respect to $\sigma$ is nonzero at $\left(\beta, \bar{u}_{1}, \sigma^{*}, \delta, \kappa\right)$. (Subsection 3.3 shows that this is true for small $\delta \geq 0$ and small $\kappa>0$.) Up to a nonzero multiple, its value at $\sigma_{*}$ is given by the Melnikov integral

$$
M=\int_{-\infty}^{\infty} \psi_{*}(\xi)^{\top} F_{\sigma}\left(X_{*}(\xi), \sigma_{*}\right) d \xi
$$

which is therefore nonzero. It is easy to check that

$$
C X_{*}^{\prime}(\xi)=-F_{\sigma}\left(X_{*}(\xi), \sigma_{*}\right) .
$$

Since $M$ is nonzero, it follows that $\frac{d D}{d \lambda}(0) \neq 0$, so 0 is a simple zero of the Evans function.

Formula (5.2) is correct despite the 0 eigenvalues at the end states; see [8]. Also, (5.3) is indeed the derivative of the separation function despite the 0 eigenvalues, because the manifolds being connected (the line of equilibria $\left(0,0, u_{2}, 0\right)$ and the equilibrium $\left.(0,0,1,0)\right)$ are independent of $\sigma$; see [20] for a similar case.

A similar discussion holds for $\kappa=0$.

5.3. Numerics for $\kappa=0$. For fixed $\left(\beta, \bar{u}_{1}, \delta\right)$ and $\kappa=0$, the traveling wave system is (3.15)-(3.17). Following the previous subsection, we write the traveling wave as $X_{*}(\xi)=$ $\left(u_{1 *}, u_{1 *}^{\prime}, u_{2 *}\right)(\xi)$. In our numerics we take $\bar{u}_{1}=0$. Then the complex number $\lambda$ is an eigenvalue of $L$ on $\mathcal{E}_{\alpha}$ provided the the following first-order system has a solution in $\mathcal{E}_{\alpha}^{3}$ :

$$
X_{\xi}=(B(\xi)+\lambda C) X=\left(\begin{array}{ccc}
0 & 1 & 0 \\
\lambda-u_{2 *}(\xi) \rho^{\prime}\left(u_{1 *}(\xi)\right)+\delta & -\sigma & -\rho\left(u_{1 *}(\xi)\right) \\
\frac{\beta}{\sigma} u_{2 *}(\xi) \rho^{\prime}\left(u_{1 *}(\xi)\right) & 0 & \frac{\lambda}{\sigma}+\frac{\beta}{\sigma} \rho\left(u_{1 *}(\xi)\right)
\end{array}\right) X
$$

The eigenfunction $\tilde{Y}$ is given by the first and third components of $X$.

We have

$$
(B( \pm \infty)+\lambda C)=\left(\begin{array}{ccc}
0 & 1 & 0 \\
\lambda+\delta & -\sigma & 0 \\
0 & 0 & \frac{\lambda}{\sigma}
\end{array}\right)
$$

The eigenvalues are

$$
\frac{\lambda}{\sigma} \quad \text { and } \quad \mu_{ \pm}(\sigma, \delta, \lambda)=-\frac{\sigma}{2} \pm\left(\frac{\sigma^{2}}{4}+\lambda+\delta\right)^{\frac{1}{2}} .
$$


For $\operatorname{Re} \lambda \geq \max \left(-\frac{1}{2} \nu_{-},-\frac{1}{2} \nu_{+}\right),(5.4)$ has linear independent solutions $X^{1}(\xi, \lambda)$ and $X^{2}(\xi, \lambda)$ such that $e^{\alpha_{-} \xi} X^{i}(\xi, \lambda), i=1,2$, is bounded for $\xi \leq 0$; they correspond to the eigenvalues $\frac{\lambda}{\sigma}$ and $\mu_{+}(\sigma, \delta, \lambda)$, which have real part small negative or larger. Similarly, (5.4) has, up to scalar multiplication, a unique solution $X^{3}(\xi, \lambda)$ such that $e^{\alpha+\xi} X^{3}(\xi)$ is bounded for $\xi \geq 0$. It corresponds to the eigenvalue $\mu_{-}(\sigma, \delta, \lambda)$, which has a large negative real part. These solutions can be chosen to depend analytically on $\lambda$. The Evans function $D(\lambda)$ is defined for $\operatorname{Re} \lambda \geq \max \left(\omega_{-}, \omega_{+}\right)<0$ by

$$
D(\lambda)=\operatorname{det}\left(X^{1}(0, \lambda), X^{2}(0, \lambda), X^{3}(0, \lambda)\right) .
$$

It is analytic, and it is 0 if and only if $\lambda$ is an eigenvalue of $L$ on $\mathcal{E}_{\alpha}$. The algebraic multiplicity of $\lambda$ as an eigenvalue of $L$ equals its multiplicity as a zero of $D$.

Up to scalar multiplication, there is unique solution $\psi(\xi, \lambda)$ of the adjoint equation $\psi_{\xi}=$ $-(B(\xi)+\lambda C)^{\top} \psi$ such that $e^{-\alpha_{+} \xi} \psi(\xi)$ is bounded on $\xi \leq 0$. It corresponds to the eigenvalue $-\mu_{-}(\sigma, \delta, \lambda)$ of $-(B( \pm \infty)+\lambda C)^{\top}$, which has a large positive real part. The Evans function can be equivalently defined as $D(\lambda)=\psi(0, \lambda)^{\top} X^{3}(0, \lambda)$. (More commonly, one uses $\bar{\lambda}$ instead of $\lambda$ in the definition of the adjoint equation, and $\bar{\psi}(0, \lambda)^{\top} X^{3}(0, \lambda)$ in the definition of the Evans function, but the two definitions of the Evans function are equivalent.)

For the traveling wave we use linear approximations on $(-\infty,-k]$ and on $[k, \infty)$, and a numerically computed approximation on $[-k, k]$.

To approximate $D(\lambda)$, choose $-k_{\ell}<0<k_{r}$. Let $X\left(k_{r}\right)=\left(1, \mu_{-}, 0\right)$, which is the eigenvector of $B( \pm \infty)+\lambda C$ for the eigenvalue $\mu_{-}$, and solve $X_{\xi}=(B(\xi)+\lambda C) X$ backward to obtain $X(0)$; let $\psi\left(-k_{\ell}\right)=\left(\lambda+\delta, \mu_{-}, 0\right)$, which is the eigenvector of $-(B( \pm \infty)+\lambda C)^{\top}$ for the eigenvalue $-\mu_{-}$, and solve $\psi_{\xi}=-(B(\xi)+\lambda C)^{T} \psi$ forward to obtain $\psi(0)$. Then $D(\lambda)$ is approximately $\psi(0)^{\top} X(0)$. One computes $D(\lambda)$ on an appropriate large closed curve $\Gamma$ that surrounds 0 . From the analyticity of $D$, if the winding number is 1 , the only zero of the Evans function inside $\Gamma$ is 0 . This is numerical evidence that the only zero of the Evans function in $\operatorname{Re} \lambda \geq 0$ is 0 .

A drawback of this approach is that computed values of $D(\lambda)$ have enormous modulus (because of the exponential growth of $X(\xi)$ and $\psi(\xi)$ ), and the curve $D(\lambda)$ can exhibit numerous twists (because of the behavior of the imaginary parts of $X(\xi)$ and $\psi(\xi)$ as they change enormously). To correct these effects, one can first define $\tilde{X}(\xi)=e^{-\mu_{-}\left(\xi-k_{r}\right)} X(\xi)$, let $\tilde{X}\left(k_{r}\right)=\left(1, \mu_{-}, 0\right)$, note that $\tilde{X}_{\xi}=\left(B(\xi)+\lambda C-\mu_{-} I\right) \tilde{X}$, and solve this equation backward to obtain $\tilde{X}(0)$. Similarly, one can define $\tilde{\psi}(\xi)=e^{\mu_{-}\left(\xi+k_{\ell}\right)} \psi(\xi)$, let $\tilde{\psi}\left(-k_{\ell}\right)=\left(\lambda+\delta, \mu_{-}, 0\right)$, note that $\tilde{\psi}_{\xi}=\left(B(\xi)+\lambda C+\mu_{-} I\right)^{\top} \tilde{\psi}$, and solve this equation forward to obtain $\tilde{\psi}(0)$; finally one defines $\tilde{D}(\lambda)$ to be $\tilde{\psi}(0)^{\top} \tilde{X}(0)$. Since $\tilde{D}(\lambda)=e^{\mu_{-}\left(k_{r}+k_{\ell}\right)} D(\lambda)$, the two functions have the same winding number about 0 on $\Gamma$.

We now show some computational results with $\left(\beta, \bar{u}_{1}, \kappa\right)=(1,0,0)$.

The computed curve in the $\delta \sigma$-plane for which traveling waves exist is shown in Figure 5.1 .

For point $A$ in Figure 5.1, we computed $\tilde{D}(\lambda)$ on several curves consisting of a right halfcircle centered at $z_{0}=-0.01$, together with the vertical diameter of that circle. The numbers $k_{\ell}$ and $k_{r}$ were increased until the desired precision was achieved. Figures 5.2 and 5.3 show the images when the radius is 1 and 100 respectively. The winding number about 0 is 1 in both cases.

For point $B$ in Figure 5.1, the image of the same curve with radius 1 is shown in Figure 5.4. The winding number is 2 , because the Evans function has a positive real root. The new 


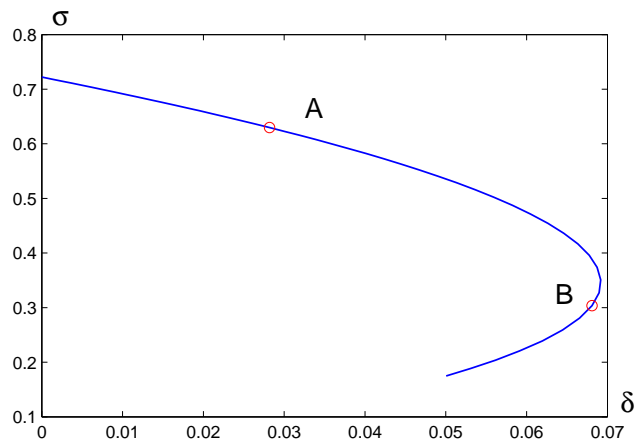

FiguRE 5.1. For the system (1.1)-(1.2) with $\left(\beta, \bar{u}_{1}, \kappa\right)=(1,0,0)$, pairs $(\delta, \sigma)$ for which traveling waves exist. Compare Figure 2.1.

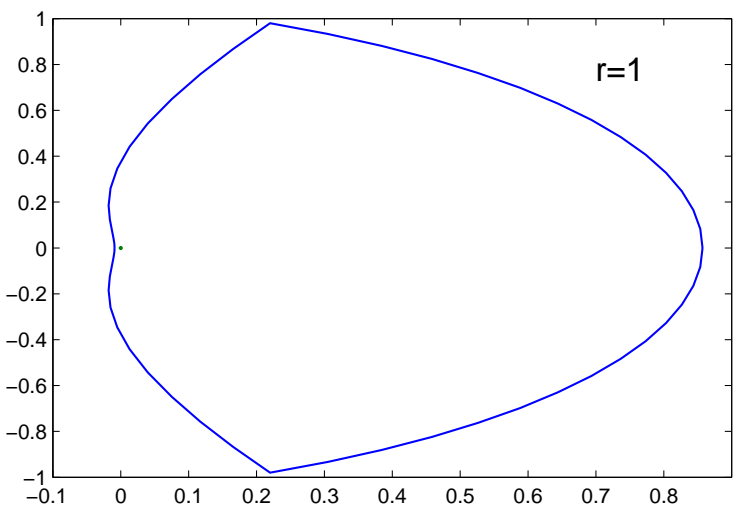

Figure 5.2. For point $A$ in Figure 5.1, the image of $\tilde{D}(\lambda)$ on a right half-circle of radius 1 centered at $z_{0}=-0.01$.

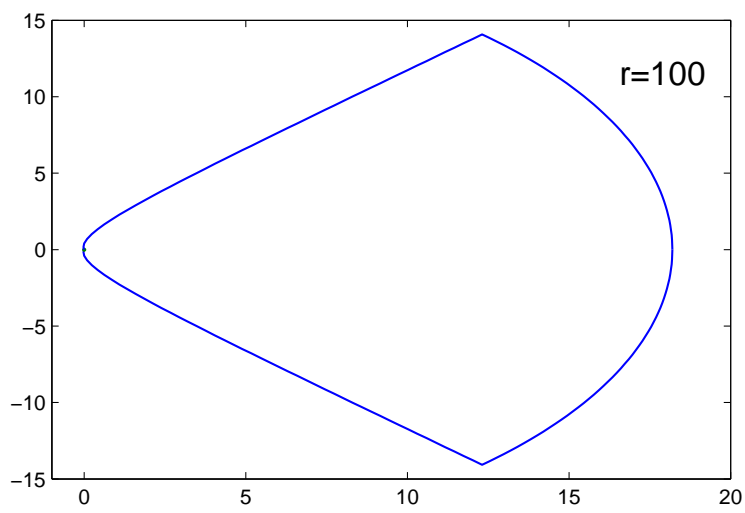

Figure 5.3. For point $A$ in Figure 5.1, the image of $\tilde{D}(\lambda)$ on a right half-circle of radius 100 centered at $z_{0}=-0.01$. 
root, which causes instability of the traveling wave, crosses the imaginary axis into the right half-plane at the turning point of the curve in Figure 5.1.

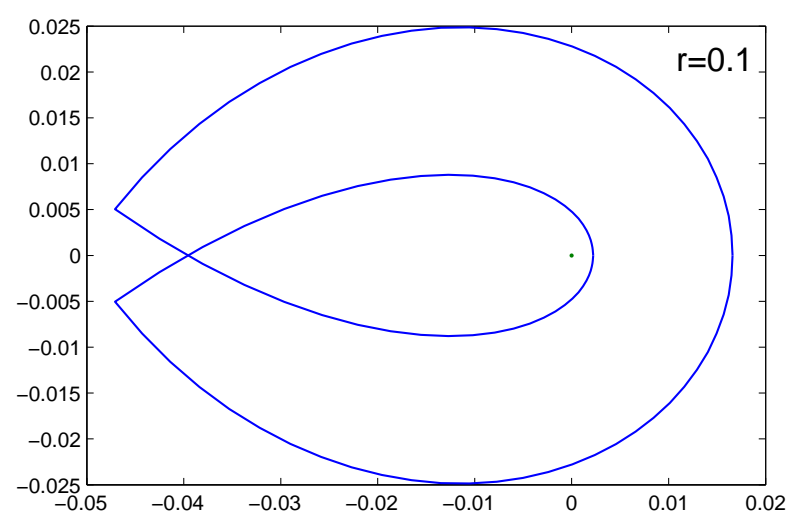

Figure 5.4. For point $B$ in Figure 5.1, the image of $\tilde{D}(\lambda)$ on a right half-circle of radius 1 centered at $z_{0}=-0.01$.

\section{REFERENCES}

[1] Bayliss, A., and Matkowsky, B., Fronts, relaxation oscillations, and period doubling in solid fuel combustion, J. Comput. Phys. 71 (1987), 147-168.

[2] Balasuriya, S., Gottwald, G., Hornibrook, J., and Lafortune, S., High Lewis number combustion wavefronts: a perturbative Melnikov analysis, SIAM J. Appl. Math. 67 (2006-07), 464-486

[3] Berestycki, H., Nicolaenko, B., and Scheurer, B., Traveling wave solutions to combustion models and their singular limits, SIAM J. Math. Anal. 16 (1985), 1207-1242.

[4] Billingham, J., Phase plane analysis of one-dimensional reaction diffusion waves with degenerate reaction terms, Dyn. Stab. Syst. 15 (2000), 23-33.

[5] Giovangigli, V., Nonadiabatic plane laminar flames and their singular limits, SIAM J. Math. Anal. 21 (1990), 1305-1325.

[6] Ghazaryan, A., Nonlinear stability of high Lewis number combustion fronts, Indiana Univ. Math. J. 58 (2009), 181-212.

[7] Ghazaryan, A., and Jones, C. K. R. T., On the stability of high Lewis number combustion fronts, Discrete Contin. Dyn. Syst. 24 (2009), 809-826.

[8] Ghazaryan, A., Latushinin, Y., Schecter, S., and De Souza, A. J., Stability of gasless combustion fronts in one-dimensional solids, Arch. Ration. Mech. Anal. 198 (2010), 981-1030.

[9] Ghazaryan, A., Latushkin, Y., and Schecter, S., Stability of traveling waves for degenerate systems of reaction diffusion equations, Indiana Univ. Math. J. 60 (2011), 443-472.

[10] Ghazaryan, A., Latushrin, Y., and Schecter, S., Stability of traveling waves for a class of reactiondiffusion systems that arise in chemical reaction models, SIAM J. Math. Anal. 42 (2010), 2434-2472.

[11] Gubernov, V. V., Mercer, G. N., Sidhu, H. S., and Weber, R. O., Evans function stability of combustion waves, SIAM J. Appl. Math. 63 (2003), 1259-1275.

[12] Gubernov, V. V., Sidhu, H. S., and Mercer, G. N., The effect of ambient temperature on the propagation of nonadiabatic combustion waves, J. Math. Chem. 37 (2005), 149-162.

[13] Jones, C. K. R. T., Geometric singular perturbation theory, Dynamical Systems (R. Johnson, ed.), Lecture Notes in Mathematics 1609, Springer, 1995.

[14] Kato, T., Perturbation theory for linear operators, second edition, Grundlehren der Mathematischen Wissenschaften, Band 132, Springer-Verlag, Berlin-New York, 1976.

[15] LOGAK, E., Mathematical analysis of a condensed phase combustion model without ignition temperature, Nonlinear Anal. 28 (1997), 1-38. 
[16] Logak, E., and Loubeau, V., Travelling wave solutions to a condensed phase combustion model, Asymptotic Anal. 12 (1996), 259-294.

[17] Marion, M., Qualitative properties of a nonlinear system for laminar flames without ignition temperature, Nonlinear Anal. 9 (1985), 1269-1292.

[18] Roques, L., Study of the premixed flame model with heat losses: The existence of two solutions, European J. Appl. Math. 16 (2005), 741765.

[19] Sandstede, B., Stability of travelling waves, in Handbook of Dynamical Systems, Vol. 2, 983-1055, North-Holland, Amsterdam, 2002.

[20] Schecter, S., The saddle-node separatrix-loop bifurcation, SIAM J. Math. Anal. 18 (1987), 1142-1156.

[21] Simon, P., Merkin, J., and Scott, S., Bifurcations in non-adiabatic flame propagation models, Focus on Combustion Research (2006), 315-357.

[22] VARAs, F. and VegA, J., Linear stability of a plane front in solid combustion at large heat of reaction, SIAM J. Appl. Math. 62 (2002), 1810-1822.

\section{Appendix A. NoRmally hyperbolic INVARIANT MaNifoldS}

The tangent space of $\mathbb{R}^{n+m}$ at $p \in \mathbb{R}^{n+m}, T_{p} \mathbb{R}^{n+m}$, is just a copy of $\mathbb{R}^{n+m}$, but the vectors in $\mathbb{R}^{n+m}$ are thought of as having tails at $p$. An element of $T_{p} \mathbb{R}^{n+m}$ is just a pair $(p, v)$ with $v \in \mathbb{R}^{n+m}$. The tangent bundle of $\mathbb{R}^{n+m}, T \mathbb{R}^{n+m}$, is the union of the spaces $T_{p} \mathbb{R}^{n+m}$ over all $p$. More precisely, $T \mathbb{R}^{n+m}=\mathbb{R}^{n+m} \times \mathbb{R}^{n+m}$; an element of $T \mathbb{R}^{n+m}$ is just a pair $(p, v)$. If $N$ is a subset of $\mathbb{R}^{n+m}, T \mathbb{R}^{n+m} \mid N$ is the union over all $p \in N$ of the $T_{p} \mathbb{R}^{n+m}$, i.e., $T \mathbb{R}^{n+m} \mid N=N \times \mathbb{R}^{n+m}$.

Now let $N$ be a smooth submanifold of $\mathbb{R}^{n+m}$ of dimension $m$. $T_{p} N$ is just the $m$ dimensional subspace of $T_{p} \mathbb{R}^{n+m}$ consisting of vectors tangent to $N$ at $p$. The tangent bundle of $N, T N$, is the union over all $p \in N$ of the spaces $T_{p} N$. More precisely,

$$
T N=\left\{(p, v) \in \mathbb{R}^{n+m} \times \mathbb{R}^{n+m}: p \in N \text { and } v \text { is tangent to } N \text { at } p\right\} .
$$

$T N$ is a subbundle of $T \mathbb{R}^{n+m} \mid N$. In general to define a $k$-dimensional subbundle $B$ of $T \mathbb{R}^{n+m} \mid N$, choose, for each $p \in N$, a $k$-dimensional subspace $B_{p}$ of $\mathbb{R}^{n+m}$; then $B=\{(p, v)$ : $p \in N$ and $v \in B_{p}$. The subspace $B_{p}$ must depend smoothly on $p$, i.e., $B$ must be a smooth submanifold of $T \mathbb{R}^{n+m}$ of dimension $k+m$. Each space $B_{p}$ is a fiber of $B$, and the division of $B$ into fibers is called a fibration.

Let $B$ and $C$ be two subbundles of $T \mathbb{R}^{n+m} \mid N$. We write $T \mathbb{R}^{n+m} \mid N=B \oplus C$ if for each $p \in N, T_{p} \mathbb{R}^{n+m}=B_{p} \oplus C_{p}$. This is called a splitting of $T \mathbb{R}^{n+m} \mid N$.

If $g: \mathbb{R}^{n+m} \rightarrow \mathbb{R}^{n+m}$ is a smooth function, then its derivative at $p, D g(p)$ is an $(n+m) \times$ $(n+m)$ matrix that induces a linear map from $T_{p} \mathbb{R}^{n+m}$ to $T_{g(p)} \mathbb{R}^{n+m}$, given by $D g(p) v=w$.

Let $\dot{p}=f(p)$ be a differential equation on $\mathbb{R}^{n+m}$ (dot is derivative with respect to $t$ ), and let $\phi^{t}$ be the flow (i.e., $\phi^{t}(q)$, with $q$ fixed, is the solution of the initial value problem $\dot{p}=f(p), p(0)=q)$.

Let $N$ be a compact submanifold or submanifold with boundary of $\mathbb{R}^{n+m}$ that is inflowing invariant under $\dot{p}=f(p)$, i.e., at points $p$ on the boundary, $f(p)$ points into the interior of $N$. $N$ is called a normally hyperbolic invariant manifold if there is a splitting $T \mathbb{R}^{n+m} \mid N=$ $S \oplus U \oplus T N$, such that under $D \phi^{t}$, as $t$ increases, all vectors in $S$ shrink at a faster exponential rate than any vector in $T N$, and as $t$ decreases, all vectors in $U$ shrink at a faster exponential rate than any vector in $T N$. There are less restrictive definitions, but this one suffices for our purposes. The spaces $S_{p}$ (respectively $U_{p}$ ) are called stable (respectively unstable) fibers.

The requirement that $N$ be inflowing invariant is easily relaxed; for example, one has normally invariant manifolds of equilibria. We will ignore this technicality. 
Normally hyperbolic invariant manifolds have stable and unstable manifolds with flowpreserved fibrations, and the whole structure persists under perturbation. This structure is most easily described in local coordinates.

Let $\dot{p}=f(p, \epsilon)$ be a differential equation on $\mathbb{R}^{n+m}$ with parameter $\epsilon$, and let $\phi_{\epsilon}^{t}$ be the flow (i.e., $\phi_{\epsilon}^{t}(q)$, with $\epsilon$ and $q$ fixed, is the solution of the initial value problem $\dot{p}=f(p, \epsilon)$, $p(0)=q)$. Let $N_{0}$ be a normally hyperbolic invariant manifold for $\dot{p}=f(p, 0)$ of dimension $m$, and suppose the fibers of $S$ (respectively $U$ ) have dimension $k$ (respectively $l$ ), with $k+l=n$. Near a point of $N_{0}$ one can choose $\epsilon$-dependent Fenichel coordinates $p=(x, y, z, \epsilon) \in \mathbb{R}^{k} \times$ $\mathbb{R}^{l} \times \mathbb{R}^{m} \times \mathbb{R}$ such that, for small $\epsilon, \dot{p}=f(p, \epsilon)$ becomes

$$
\begin{aligned}
& \dot{x}=A(x, y, z, \epsilon) x, \\
& \dot{y}=B(x, y, z, \epsilon) y, \\
& \dot{z}=h(z, \epsilon)+x^{T} C(x, y, z, \epsilon) y ;
\end{aligned}
$$

the matrices $A$ and $B$ are $k \times k$ and $l \times l$ respectively, and $C$ is an $m$-tuple of $k \times l$ respectively. One cannot assume that $A(0,0, z, 0)$ has eigenvalues with negative real part or that $B(0,0, z, 0)$ has eigenvalues with positive real part, since the exponential convergence of vectors under the linearized flow need only occur as $t \rightarrow \pm \infty$. Nevertheless, this is true in the examples below.

We list some facts and terminology.

(1) For each $\epsilon$, the subspaces $y=0, x=0$, and their intersection are invariant. For fixed $\epsilon$, the set $\{(x, y, z) \mid x=0$ and $y=0\}$ (dimension $m$ ) corresponds to part of a normally hyperbolic invariant manifold $N_{\epsilon}$; the set $y=0$ (dimension $m+k$ ) corresponds to part of the stable manifold of $N_{\epsilon}, W^{s}\left(N_{\epsilon}\right)$; and the set $x=0$ (dimension $m+l$ ) corresponds to part of the unstable manifold of $N_{\epsilon}, W^{u}\left(N_{\epsilon}\right)$.

(2) If $(x(t), 0, z(t))$ is a solution in $W^{s}\left(N_{\epsilon}\right)$, then $(0,0, z(t))$ is a solution in $N_{\epsilon}$; and if $(0, y(t), z(t))$ is a solution in $W^{u}\left(N_{\epsilon}\right)$, then $(0,0, z(t))$ is again a solution in $N_{\epsilon}$. Thus each solution in $W^{s}\left(N_{\epsilon}\right)$ (respectively $W^{u}\left(N_{\epsilon}\right)$ ) approaches exponentially a solution in $N_{\epsilon}$ as time increases (respectively decreases).

(3) Given a point $p=\left(0,0, z_{0}\right)$ in $N_{\epsilon}$, the stable (respectively unstable) fiber of $p$ is the set of all points $\left(x, 0, z_{0}\right)$ (dimension $k$ ) (respectively $\left(0, y, z_{0}\right)$ (dimension $\left.l\right)$ ). For each $t$, the time- $t$ map of the flow takes fibers to fibers; in this sense, the fibration is flow-invariant. Solutions that start in the stable (respectively unstable) fiber of $p$ approach the solution that starts at $p$ exponentially at $t$ increases (respectively decreases).

Examples:

(1) A hyperbolic equilibrium is a hyperbolic invariant manifold of dimension 0.

(2) Suppose $N_{0}$ is a compact manifold of equilibria of dimension $m$, and each equilibrium in $N_{0}$ has $k$ eigenvalues with negative real part and $l$ eigenvalues with positive real part. Then $N_{0}$ is a compact normally hyperbolic invariant manifold. The stable (respectively unstable) fiber of a point $p$ in $N_{0}$ is just its stable (respectively unstable) manifold. In (A.3), $h(z, 0) \equiv 0$.

(3) Fast-slow systems. Consider the system

$$
\begin{aligned}
\dot{w} & =f(w, z, \epsilon), \\
\dot{z} & =\epsilon g(w, z, \epsilon),
\end{aligned}
$$


with $w \in \mathbb{R}^{n}, z \in R^{m}$, and $0 \leq \epsilon<\epsilon_{0}$. System (A.4)-(A.5) is a fast-slow system; $w$ is the fast variable and $z$ is the slow variable. The fast limit is (A.4)-(A.5) with $\epsilon=0$. If 0 is a regular value of $f$, then $\{(w, z): f(w, z, 0)=0\}$ is a manifold of dimension $m$ called the slow manifold. The slow manifold is the set of equilibria of the fast system. Let $N_{0}$ be a compact subset of the slow manifold that is a manifold with boundary of dimension $m . N_{0}$ is normally hyperbolic if there are numbers $k$ and $l$ with $k+l=n$ such that at each point $(w, z)$ of $N_{0}, D_{z} f(w, z, 0)$ has $k$ eigenvalues with negative real part and $l$ eigenvalues with positive real part. By the Implicit Function Theorem, $N_{0}$ can be described as $w=\chi(z, 0)$. Then for $\epsilon>0$ small there is a normally hyperbolic invariant manifold $N_{\epsilon}$ near $N_{0}$, given by $w=\chi(z, \epsilon)$.

The name "geometric singular perturbation theory" comes from the fact that after a rescaling of time, (A.4)-(A.5) can be rewritten as

$$
\begin{aligned}
\epsilon \dot{w} & =f(w, z, \epsilon), \\
\dot{z} & =g(w, z, \epsilon),
\end{aligned}
$$

which is a singularly perturbed system.

In Example 3, $N_{\epsilon}$ is given by an expansion $w=\chi_{0}(z)+\epsilon \chi_{1}(z)+\ldots$, and the system restricted to $N_{\epsilon}$, with coordinate $z$, is given by

$$
\dot{z}=\epsilon g\left(\chi_{0}(z)+\epsilon \chi_{1}(z)+\ldots, z, \epsilon\right)=\epsilon g\left(\chi_{0}(z), z, 0\right)+\mathcal{O}\left(\epsilon^{2}\right) .
$$

Thus, after division by $\epsilon$, system (A.4)-(A.5) restricted to $N_{\epsilon}$ is given by $\dot{z}=g\left(\chi_{0}(z), z, 0\right)+$ $\mathcal{O}(\epsilon)$. The differential equation $\dot{z}=g\left(\chi_{0}(z), z, 0\right)$ is called the slow equation.

Department of Mathematics, Miami University, Oxford, Ohio 45056, USA, +1-513-529-0582

E-mail address: ghazarar@miamioh.edu

Department of Mathematics, North Carolina State University, Box 8205, Raleigh, North Carolina 27695, USA, +1-919-515-6533

E-mail address: schecter@ncsu.edu

Department of Applied Analysis and Computational Mathematics, EÖtvös Loránd University, Budapest, Hungary, +36-1-272-2500

E-mail address: simonp@cs.elte.hu 